\title{
On Uniqueness in the Inverse Problem for Transversely Isotropic Elastic Media with a Disjoint Wave Mode
}

\author{
Anna L. Mazzucato ${ }^{\mathrm{a}, 1}$, Lizabeth V. Rachele ${ }^{\mathrm{b}, *, 2}$ \\ ${ }^{a}$ Department of Mathematics, Pennsylvania State University, University Park, PA 16802, USA



\begin{abstract}
We study general anisotropic elastic media that have a disjoint wave mode, and extend results from microlocal analysis to describe the propagation of singularities for the disjoint mode. Applying these results to the study of the dynamic inverse problem, we show that traction-displacement surface measurements uniquely determine the travel time between boundary points for the disjoint mode. We conclude that two of the five material parameters describing transversely isotropic elastodynamics with ellipsoidal slowness surfaces and a disjoint mode are partially determined by surface measurements. Our approach is well suited to inhomogeneous materials and applying microlocal analysis to the inverse problem.
\end{abstract}

Key words: Parameter identification, Dirichlet-to-Neumann map, elastodynamics, transverse isotropy, ellipsoidal slowness surface, propagation of singularities, microlocal analysis

2000 MSC: 35R30, 74Bxx, 35Sxx, 35A21, 35Lxx

\section{Introduction and Main Results}

In this paper we apply microlocal analysis to the study of wave propagation in inhomogeneous, anisotropic elastic media. Our main interest is the inverse problem for elastodynamics, the problem of determining the material properties of a bounded elastic object, given certain data which is usually collected remotely. There are important applications of the inverse problem in medical imaging and seismic tomography, for example. (See, e.g., [1], [2], [3], $[4],[5]$.

We model elastodynamics in terms of the system (3) of linear partial differential equations on a bounded domain $\Omega \subset \mathbb{R}^{3}$. The coefficients of these differential equations, in the form of a scalar function $\rho$ and a rank-4 tensor field C, represent the density and elastic properties of the object. The data for the inverse problem are modeled by the dynamic Dirichlet-to-Neumann (DN) map, which gives the correspondence between surface displacement and surface traction.

* Corresponding author.

Email addresses: mazzucat@math.psu.edu (Anna L. Mazzucato), rachel@rpi.edu (Lizabeth V. Rachele).

1 The first author was partially supported by NSF grant DMS-0405803.

2 The second author was supported by NSF grant DMS-0340530. 
We consider the uniqueness question, that is, whether the DN map uniquely determines the material parameters $\rho, \mathbf{C}$ in the interior of $\Omega$. On the one hand, a negative answer to this question is suggested by the observation that for general anisotropic elastic media the parameters may be determined only up to coordinate changes fixing the surface of the object. We refer to this as the "natural obstruction". In [6] we show that the "natural obstruction" follows from the representation of the equations of motion in a covariant form that preserves the underlying physics (cf. [7]).

It is not clear, though, whether this obstruction arises when the class of anisotropic media is restricted to transverse isotropy. Transverse isotropy is characterized by the property that at each point the elastic response of the medium is isotropic in the plane orthogonal to the so-called fiber direction. We consider media in which the fiber direction may vary smoothly from point to point. Hexagonal crystals [8] and biological tissues, such as muscle, exhibit transversely isotropic behavior. Also, it is not known for general anisotropic elastodynamics whether there are obstructions to uniqueness other than the natural obstruction.

Here we show that for certain classes of transversely isotropic media two of the six material parameters are partially determined by the dynamic DN map. This is a consequence of our main result that for general anisotropic elastodynamics with a disjoint wave mode the travel time along rays for that mode is uniquely determined by the DN map. (We say that the system for elastodynamics has a disjoint wave mode if the corresponding sheet of the slowness surface is always distinct from the other sheets, or equivalently, if the corresponding eigenvalue of the determinant of the principal symbol is always distinct from the other eigenvalues.) For transverse isotropy the disjoint-mode condition does not appear restrictive; in the case of the 204 transversely isotropic materials listed in [9, pages 39-49] for which elasticity parameters have been computed completely, we find in [10] that each has a disjoint mode.

Sylvester and Uhlmann [11] prove that the "natural obstruction" is the only obstruction to uniqueness in the case of scalar, anisotropic models for acoustic wave propagation. In particular, they show that the travel time of the wave through the object is uniquely determined by the associated DN map. Uniqueness also holds for isotropic elastodynamics [12], [13], [14], and, up to the natural obstruction, for isotropic media with residual stress [15], [16]. Hansen and Uhlmann [16] use a microlocal analytic approach to the study of reflection of singularities to prove that the scattering relation at the surface is determined by the Dirichlet-to-Neumann map, which extends Rachele's result [15] to the case that caustics are present. In addition, Robertson shows that the Dirichlet-to-Neumann map uniquely determines the residual stress tensor and one of the Lamé parameters at the boundary [17], and establishes uniqueness in the interior [18] for the linearized problem using Man's model [19] for residual stress. Isakov, Nakamura, and Wang [20], [21], consider the unique continuation property for solutions of the Cauchy problem (see also [22]), and use it to study the inverse problem of identifying inclusions or cavities in the body. Lin and Wang [23] exploit Carleman estimates and unique continuation to prove unique identification of the density by a single boundary measurement. Also, recently Ivanov, Man, and Nakamura, using the model for residual stress by Man [19], have shown that traction-displacement measurements uniquely determine the residual stress and its gradient at the surface of an unbounded, layered medium, and near the surface if the stress is in diagonal form [24], while Rachele [15] proves unique determination of the density, isotropic elasticity parameters, and the residual stress tensor at the surface in the case of a bounded elastic object, using Hoger's model [25] , [26] for residual stress. Finally, in the case of the static inverse problem for transversely isotropic elasticity Nakamura, Tanuma, and Uhlmann [27] show that material properties are uniquely determined by surface measurements. (Here we consider the general dynamic problem, which cannot be reduced to the static case.)

To address the question of uniqueness in the inverse problem we first apply a microlocal decoupling of the system of equations for elastodynamics to show that the travel time of a disjoint wave mode through an anisotropic elastic object is determined by the Dirichlet-to-Neumann map. Then, in the case that the rays for the disjoint mode consist of geodesic segments of Riemannian metrics, we employ boundary rigidity results [28], [29], [30], [31], which hold under geometric conditions on the elastic object, to establish uniqueness of the metrics (up to coordinate changes) once the geodesic distances (i.e., travel times) between points on the surface are determined. Finally, for transverse isotropy we show that from knowledge of the metrics we can recover information about some of the parameters. We can only study travel times for the disjoint wave mode, consequently study uniqueness for only a few parameters, as propagation of singularities for the system (3), which in general is not strictly hyperbolic nor of real principal type, is not yet well understood. 
Our main result is the following. (See Theorem 1 in Section 3.)

Suppose that $\mathbb{R}^{3} \backslash \Omega$ is diffeomorphic to the exterior of a ball in $\mathbb{R}^{3}$, and let $P_{\rho, \mathrm{C}}$ be any system (3) of differential operators for general anisotropic elastodynamics on $\Omega \times[0, T]$ with density $\rho>0$, elasticity tensor $\mathbf{C}$ strongly elliptic on $\Omega$, and $\rho, \mathbf{C}$ smooth on $\bar{\Omega}$. Suppose that one of the eigenvalues of the principal symbol of $P_{\rho, \mathbf{C}}$ is always distinct from the others. Let $P_{\widetilde{\rho}, \tilde{\mathbf{C}}}$ be another operator satisfying the same conditions as $P_{\rho, \mathbf{C}}$, and suppose that the parameters $\rho, \tilde{\rho}$ and $\mathbf{C}, \tilde{\mathbf{C}}$ agree to infinite order at the boundary of $\Omega$. Suppose that the dynamic Dirichlet-to-Neumann maps agree on $\partial \Omega \times(0, T)$ :

$$
\Lambda_{\rho, \mathbf{C}}=\Lambda_{\tilde{\rho}, \tilde{\mathbf{C}}}
$$

where $T$ is larger than the travel time with respect to the disjoint modes between any two boundary points of $\Omega$. Then the travel time along any disjoint-mode bicharacteristic that connects boundary points is determined by the Dirichlet-to-Neumann map.

In fact, we also show that the exit point and direction of any disjoint-mode bicharacteristic through $\Omega$ are uniquely determined by the Dirichlet-to-Neumann map. That is, the scattering relation is uniquely determined.

The assumption that $\mathbb{R}^{3} \backslash \Omega$ is diffeomorphic to the exterior of a ball in $\mathbb{R}^{3}$ is a technical condition and is not needed in the case of transversely isotropic media. We emphasize that all transversely isotropic elastic materials listed in [9, pages 39-49] have a disjoint mode. (See [10].)

Microlocal analysis of wave propagation may be seen as an extension of the classical ray-tracing approach well known in seismic exploration. The paths of wave propagation through the interior of an elastic solid may be described for general linear elastodynamics as integral curves of Hamiltonian functions, which, when the Hamiltonian is regular, are geodesics with respect to Finsler metrics [32, Chapter 1, Section 1], [33, Section 1.1], [34]. In special cases such metrics are Riemannian and the slowness surface is a union of ellipsoids. This so-called elliptic transverse isotropy has been studied, for example, by Rudzki [35], Helbig [36,37], Payton [38], Chadwick and Norris [39], Burridge, Chadwick, and Norris [40], and Bakker [41]. (See [10] for references on this topic.) In the case of elliptic transverse isotropy the system of differential equations that models the behavior of the corresponding elastic waves is more tractable due to the possibility of decoupling the system into linear, scalar wave equations for each of the wave modes. In fact, Burridge, Chadwick, and Norris [40] present the Green's tensor on $\mathbb{R}^{3}$ for homogeneous elliptic transversely isotropic elastic media in terms of fundamental solutions of scalar wave equations, and Daley and Lines [42] study general transversely isotropic media by comparing them with transversely isotropic media with ellipsoidal slowness surfaces.

Slowness surfaces arise in solving the system of elastodynamics (3) by the method of characteristics. The characteristic condition is given by the vanishing of the determinant of the principal symbol of the operator. Each sheet $1=\lambda(x, s)$ of the slowness surface corresponds to a different factor $\tau^{2}-\lambda(x, \xi)$ of the determinant. Here $\lambda(x, \xi)$ is the square of the speed of propagation of a wave front with normal direction $\xi$ at $x \in \Omega$ for a particular wave mode. If the factors of the determinant are polynomial in the covariable (or wave normal) $\xi$, then these factors are symbols of second-order, scalar, linear differential operators, and the characteristic curves for the entire system are characteristics for such operators. In particular, if the sheets of the slowness surface have the form $1=s^{t} g^{-1} s$, where $g$ is a Riemannian metric, then characteristics are (piecewise) geodesic segments with respect to $g$. Consequently, we say that a wave mode has geodesic wave propagation (GWP) in $\Omega$ if the corresponding sheet of the slowness surface is ellipsoidal. In addition, we say that an elastic medium has geodesic wave propagation in $\Omega$ if each mode does, that is, the determinant of the principal symbol may be factored at each $x \in \Omega$ as

$$
-\rho^{3}\left(\tau^{2}-\xi^{t} g_{1}^{-1}(x) \xi\right)\left(\tau^{2}-\xi^{t} g_{2}^{-1}(x) \xi\right)\left(\tau^{2}-\xi^{t} g_{3}^{-1}(x) \xi\right)
$$

where the $g_{i}$ are smooth, symmetric, positive-definite matrices depending only on $x$. 
A consequence of our main result in the case that the disjoint mode has geodesic wave propagation is the following corollary.

Consider anisotropic elastic media $(\Omega, \rho, \mathbf{C})$ and $(\Omega, \tilde{\rho}, \tilde{\mathbf{C}})$, each having a disjoint mode. Suppose that the disjoint eigenvalues of the determinant of the principal symbol have the form $\rho\left(\tau^{2}-\xi^{t} g(x)^{-1} \xi\right), \rho\left(\tau^{2}-\xi^{t} \widetilde{g}(x)^{-1} \xi\right)$ on $\bar{\Omega}$, where $g, \widetilde{g}$ are Riemannian metrics, and $\Omega$ is strictly convex with respect to each of the two metrics. If the dynamic Dirichlet-to-Neumann maps agree on $\partial \Omega \times(0, T):$

$$
\Lambda_{\rho, \mathbf{C}}=\Lambda_{\tilde{\rho}, \tilde{\mathbf{C}}}
$$

where $T$ is larger than the geodesic distance with respect to both $g_{2}$ and $\widetilde{g}_{2}$ between any two boundary points $\underline{x}, \bar{x} \in \partial \Omega$, then the geodesic distances with respect to $g$ and $\tilde{g}$ also agree:

$$
d_{g_{2}}(\underline{x}, \bar{x})=d_{\tilde{g}_{2}}(\underline{x}, \bar{x}) \quad \text { for all } \underline{x}, \bar{x} \in \partial \Omega .
$$

We conclude that the metrics $g, \widetilde{g}$ are determined by the Dirichlet-to-Neumann map, up to pullback by a diffeomorphism that fixes the boundary, by applying boundary rigidity results [28], [29], [30], or [31], for example. For boundary rigidity results in the 2-dimensional case we refer to [43].

We present examples (CM1) and (CM2) of anisotropic elastic media with a disjoint mode in [10]. The classes (CM1) and (CM2) consist exactly of the transversely isotropic elastic media with GWP and a disjoint mode, and so satisfy the conditions of our main result and its corollary if $\Omega$ is strictly convex with respect to each of $g, \widetilde{g}$. The conditions for (CM2) may be derived from Payton [38, p. 693-695], who shows that the quasi-shear and quasi-compressional sheets of the slowness surface are distinct when $\left(A+\mu_{T}-\mu_{L}\right)\left(B-\mu_{L}\right)\left(C+\mu_{L}\right)^{2}>0$ or $\left(A+\mu_{T}-\mu_{L}\right)\left(B-\mu_{L}\right)<0$; and that in the positive-definite, ellipsoidal case with $A+\mu_{T}>\mu_{L}$ and $B>\mu_{L}$ the pure shear sheet intersects the quasi-shear and quasi-compressional sheets only at the fiber, or is coincident with the quasi-shear sheet. See Section 2.2 for the definition of the material parameters $\mu_{L}, \mu_{T}, A, B, C$.) We refer to [10] for additional references on subclasses of transversely isotropic elastic media.

Each of the transversely isotropic materials listed in [9, pages 39-49] has a disjoint mode, and two (a form of the ceramic $\mathrm{BaTiO}_{3}$ with $\mu_{L}=43, \mu_{T}=44.5, A=121.5, B=162, C=78$, and a polymer polymethyl methacrylate (Perspex) with $\mu_{L}=2.3, \mu_{T}=2.1, A=6.0, B=11.0, C=4.8$ ) are very close to GWP. These two materials are (essentially) of type (CM2).

We observe in [10] that the metrics describing wave propagation for transversely isotropic elastic media with GWP all have the form $g^{-1}=p I+q(k \otimes k)$, where $k$ is the fiber direction, and conclude in Section 3.4 that for (CM1) and (CM2) two of the five (density and elasticity) parameters are determined by the Dirichlet-to-Neumann map (up to certain factors associated with the boundary rigidity).

We prove the main result in Section 3.2 by microlocally decoupling the equations for elastodynamics using results of Taylor [44]. This decoupling holds only when the light cone for one of the wave modes is disjoint from those associated with the other two modes. In the case that the disjoint mode has GWP the component of the displacement $w$ associated with the disjoint mode solves a scalar, linear wave equation of the form:

$$
\left(\partial_{t}^{2}-\Delta_{g}+B(x, t, D)\right) w=F
$$

where $B$ is a pseudo-differential operator of order $1, \Delta_{g}$ is the Laplace operator associated with the Riemannian metric $g$, and $F$ is a smooth remainder. Here $g$ is one of the metrics appearing in the decomposition (1) of the determinant of the principal symbol.

A similar decoupling of the system of elastodynamics for anisotropic elastic media has been used by other authors. For example, Hansen and Uhlmann decouple the equations near the boundary in studying propagation of polarization in elastodynamics with residual stress [16]. Here, we adapt the approach taken by Stolk and de Hoop, who treat seismic inverse scattering for anisotropic media in [3], under the assumption that the multiplicity of some eigenvalues is constant, at least microlocally. Our approach differs from Stolk and de Hoop's in that they decouple the system in a neighborhood of a given (but arbitrary) point and direction, while we decouple the system in a neighborhood of a bicharacteristic for $t \in[0, T], T$ not necessarily small. 
Braam and Duistermaat [45] study generic anisotropic elastic media and conjugate the operator to normal form. Nolan and Uhlmann [46] apply this result to construct microlocal parametrices. Transversely isotropic elastic media with GWP do not necessarily satisfy the conditions of Braam and Duistermaat, as the doubly characteristic set can be involutive and the Hamilton vector field associated with the determinant of the principal symbol may not have rank three. This is the case, for example, for (GWP2) media with $\mu_{L}=\mu_{T}$, which have two coincident eigenvalues. We do not know whether other anisotropic elastic media with a disjoint wave mode generically satisfy the conditions in [45].

To solve the inverse problem we assume that the material parameters are determined to infinite order at the boundary of the elastic object, and we proceed as in [11] and also [13], [14], [15], to extend the parameters to all of $\mathbb{R}^{3}$ in a smooth fashion, here in a way that preserves strong ellipticity, the property of having a disjoint mode, and, for the corollary, the GWP property for the disjoint mode. To make this smooth extension in the case of general anisotropy we assume that $\mathbb{R}^{3} \backslash \Omega$ is diffeomorphic to the exterior of a ball in $\mathbb{R}^{3}$, but in the case of transverse isotropy we do not need this assumption. We then consider the Cauchy problem for elastodynamics in $\mathbb{R}^{3}$. Given appropriate choices for initial data (with "minimal" wave front set), we show that disturbances for the disjoint mode propagate along just two bicharacteristics for $\tau^{2}-\lambda(x, \xi)$, where $\lambda(x, \xi)$ is the wave speed (squared) for the disjoint mode.

In the special case that the slowness surface is a disjoint union of ellipsoids, or equivalently the light cones for each wave mode do not intersect, the system for elastodynamics is of real principal type, and approximate solutions may be constructed via geometrical optics. (See, for example, [47], [48], [49], and [15]). In media of this type singularities propagate along disjoint geodesic segments for the metrics $g_{i}$ [50], and Rachele shows [15] that the metrics $g_{i}, i=1, \ldots, 3$, are uniquely determined by the Dirichlet-to-Neumann map, up to pullback by diffeomorphisms $\psi_{i}, i=1, \ldots, 3$ that fix the surface of the object (under the condition that caustics do not arise in the interior and there are no glancing points at the boundary). The principal example of elastic media with GWP studied in [15] is isotropic elastodynamics with residual stress. Hansen and Uhlmann [16] establish similar results for isotropic elastodynamics with residual stress in the presence of caustics.

The paper is organized as follows. We introduce the Dirichlet-to-Neumann map and the equations for elastodynamics in Section 2.1, and the conditions (CM1) and (CM2) under which there is a disjoint sheet of the slowness surface for GWP in Section 2.2. Then in Section 3 we study the inverse problem. We extend the problem to $\mathbb{R}^{3}$ in Section 3.1; decouple the disjoint wave mode in Section 3.2; prove the unique determination of the boundary distance function associated with the disjoint mode in Section 3.3; and conclude that certain material parameters are determined by the Dirichlet-to-Neumann map in Section 3.4.

\section{Preliminaries}

\subsection{The Dirichlet-to-Neumann map}

We consider a three-dimensional elastic object modeled by a bounded region $\Omega$ in $\mathbb{R}^{3}$ with smooth boundary $\partial \Omega$. The density and elastic parameters are modeled by the $C^{\infty}(\bar{\Omega})$ smooth function $\rho(x)$ and rank-4 tensor field $\mathbf{C}(x)$. The elasticity tensor in the case of general hyperelasticity has the symmetry properties

$$
\mathrm{C}_{i j k l}=\mathrm{C}_{k l i j}=\mathrm{C}_{j i k l}=\mathrm{C}_{i j l k},
$$

and is (uniformly) strongly elliptic on $\Omega$, i.e., there is a constant $c>0$ so that for any $x \in \Omega \quad \mathrm{C}_{i j k l}(x) V^{i} W^{j} V^{k} W^{l} \geq$ $c|\mathbf{V}|^{2}|\mathbf{W}|^{2}$ for all vectors $\mathbf{V}, \mathbf{W}$. It follows that the $\lambda_{i}(x, \xi)$ that appear in (9) are positive. The elasticity tensor is positive-definite on $\Omega$ if for any $x \in \Omega \quad \mathrm{C}_{i j k l}(x) E^{i j} E^{k l} \geq 0$ for any symmetric matrix $E$.

The displacement after time $t$ of a point initially at position $x$ is represented by the vector-valued function $u(x, t)=\left(u_{1}, u_{2}, u_{3}\right)$ which solves the initial-boundary-value problem

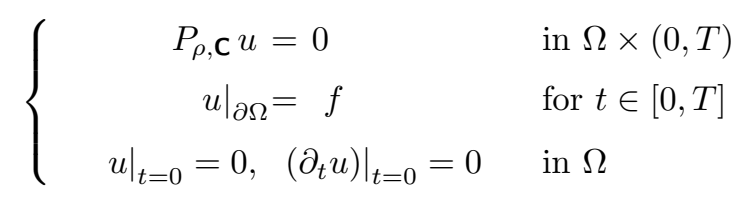


associated with the system of differential operators

$$
\left(P_{\rho, \mathbf{c}} u\right)_{i}=\rho(x) \frac{\partial^{2} u_{i}}{\partial t^{2}} \quad-\sum_{j, k, l=1}^{3} \frac{\partial}{\partial x_{j}}\left(\mathrm{C}_{i j k l}(x) \frac{\partial u_{k}}{\partial x_{l}}\right), \quad i=1,2,3,
$$

for general elastodynamics. We refer to [7], p. 369 (see also [51], Theorem III.4.1), for conditions under which existence and uniqueness holds for (2).

The Dirichlet-to-Neumann map $\Lambda_{\rho, \mathrm{C}}$ for $P_{\rho, \mathrm{C}}$ is defined in terms of solutions $u$ of the initial-boundary-value problem (2) by

$$
\left(\Lambda_{\rho, \mathbf{C}} f\right)_{i}=\sum_{j, k, l=1}^{3} \nu_{j} \mathrm{C}_{i j k l} \frac{\partial u_{k}}{\partial x_{l}} \underset{\partial \Omega \times(0, T)}{\mid}, \quad i=1,2,3,
$$

where $\nu$ is the unit outer normal vector to $\partial \Omega$. That is, the DN map gives the correspondence between the surface displacement $f$ and the surface traction $\Lambda_{\rho, \mathbf{C}} f$.

\subsection{Geodesic wave propagation for transversely isotropic elastic media}

Definition of certain combinations of the material parameters. We introduce the following combinations $A, B, C$ of the material parameters (the transverse and longitudinal Young's moduli $E_{T}, E_{L}$, Poisson's ratios $\nu_{T}, \nu_{L}$, and shear moduli $\left.\mu_{T}, \mu_{L}\right)$. Let

$$
A=\frac{E_{L} E_{T}}{2\left[E_{L}\left(1-\nu_{T}\right)-2 E_{T} \nu_{L}^{2}\right]}, \quad B=\frac{E_{L}^{2}\left(1-\nu_{T}\right)}{E_{L}\left(1-\nu_{T}\right)-2 E_{T} \nu_{L}^{2}}, \quad C=\frac{\sigma_{s i n} E_{L}\left|\nu_{L}\right| E_{T}}{E_{L}\left(1-\nu_{T}\right)-2 E_{T} \nu_{L}^{2}},
$$

where $\sigma_{\text {sin }}=\mathbf{C}\left[k, k, k^{\perp}, k^{\perp}\right] /\left|\mathbf{C}\left[k, k, k^{\perp}, k^{\perp}\right]\right|$ with $k^{\perp}$ any unit vector orthogonal to $k$. (See [10].)

We observe in [10] that the material parameters $A, B, C, \mu_{T}, \mu_{L}$ are related to components $c_{i j}$ (with respect to an orthonormal basis $\left\{e_{1}, e_{2}, e_{3}\right\}$ with $e_{3}=k$ ) of the transversely isotropic elasticity tensor $\mathbf{C}$ as follows:

$$
\mu_{T}=\left(c_{11}-c_{12}\right) / 2=c_{66}, \quad \mu_{L}=c_{44}, \quad A=\left(c_{11}+c_{12}\right) / 2, \quad B=c_{33}, \quad C=c_{13},
$$

where $c_{i j}=\mathbf{C}\left[e_{i}, e_{i}, e_{j}, e_{j}\right], \quad i, j=1,2,3 ; c_{i j}=0, i \neq j=4,5,6 ; c_{11}=c_{22}, c_{13}=c_{23}, \quad c_{44}=c_{55}=$ $\mathbf{C}\left[e_{2}, e_{3}, e_{2}, e_{3}\right], c_{66}=\mathbf{C}\left[e_{1}, e_{2}, e_{1}, e_{2}\right]$.

Positive-definiteness conditions. The elasticity tensor for transversely isotropic elastodynamics is positivedefinite if and only if

$$
\mu_{T}>0, \quad \mu_{L}>0, \quad A>0, \quad B>0, \quad A B>C^{2} .
$$

Strong ellipticity conditions Necessary and sufficient conditions for strong ellipticity of the transversely isotropic elasticity tensor are given by Merodio and Ogden $[52,(2.9),(2.12)]$ to be

$$
c_{11}>0, \quad c_{33}>0, \quad c_{44}>0, \quad c_{11}>c_{12}, \quad\left|c_{13}+c_{44}\right|<c_{44}+\sqrt{c_{11} c_{33}}
$$

in terms of components $c_{i j}$ of the elasticity tensor with respect to an orthonormal basis $\left\{e_{1}, e_{2}, e_{3}\right\}$ with $e_{3}=k$. That is, $\mathbf{C}$ is strongly elliptic if and only if

$$
\mu_{T}>0, \quad \mu_{L}>0, \quad A+\mu_{T}>0, \quad B>0, \quad \text { and } \quad\left|\mu_{L}+C\right|<\mu_{L}+\sqrt{\left(A+\mu_{T}\right) B} .
$$

Pointwise conditions so that each $\lambda(x, \xi)$ is of the form $p \pm|q|$. The factors $\tau^{2}-\lambda(x, \xi)$ in the determinant of the principal symbol of $P_{\rho, \mathbf{C}}$ have $\lambda=p \pm|q|$ with $p(x, \xi), q(x, \xi)$ polynomials in $\xi$ if and only if one of the two following conditions holds:

$$
\text { (Q1) } \quad \mu_{L}+C=0, \quad \text { i.e., } c_{13}+c_{44}=0, \quad \text { i.e., } a_{5}=0
$$




$$
\begin{aligned}
& \left(\mu_{L}+C\right)^{2}=\left(A+\mu_{T}-\mu_{L}\right)\left(B-\mu_{L}\right), \quad \text { which is equivalent to each of: } \\
& \left(c_{13}+c_{44}\right)^{2}=\left(c_{11}-c_{44}\right)\left(c_{33}-c_{44}\right), \quad \mu_{L}=\frac{\left(A+\mu_{T}\right) B-C^{2}}{A+\mu_{T}+B+2 C} .
\end{aligned}
$$

See $[40$, p. $656,(3.13),(6.13)]$, and $\left[39,(1.1),(1.2),(C 3)_{1},(P 3)_{1}\right]$ for a derivation of these conditions in terms of the components $c_{i j}$ of the elasticity tensor with respect to a special basis. The notation used in these articles is similar to that used here: $a_{1}=\left(A+\mu_{T}\right) / \rho, a_{2}=\mu_{T} / \rho, a_{3}=B / \rho, a_{4}=\mu_{L} / \rho, a_{5}=\left(C+\mu_{L}\right) / \rho$.

Pointwise conditions for geodesic wave propagation GWP. Under additional conditions each of the $\lambda(x, \xi)$ in case (Q1) is a polynomial in $\xi$, and each of the sheets of the corresponding slowness surface is ellipsoidal. (Q2) already has these properties. It follows that for (Q2), and for (Q1) under the additional conditions, wave propagation through the interior occurs along geodesic segments of the Riemannian metric given by $g^{i j}(x) \xi_{i} \xi_{j}=\lambda(x, \xi)$. We refer to wave propagation along geodesic segments as geodesic wave propagation (GWP). In fact, each term $\lambda$ in the factors $\tau^{2}-\lambda(x, \xi)$ of the determinant of the principal symbol of $P_{\rho, \mathrm{C}}$ is a polynomial in $\xi$ if and only if one of the two following conditions holds:

$$
\begin{array}{ll}
(G W P 1) & \mu_{L}+C=0, \text { and } B \leq \mu_{L} \leq A+\mu_{T} \quad \text { or } \quad A+\mu_{T} \leq \mu_{L} \leq B \\
(G W P 2) & \left(\mu_{L}+C\right)^{2}=\left(A+\mu_{T}-\mu_{L}\right)\left(B-\mu_{L}\right) .
\end{array}
$$

Examples: Special cases close to isotropy. An example of transverse isotropy with GWP is the case with both $(G W P 1)$ and (GWP2):

$$
(G W P 12) \quad \mu_{L}=A+\mu_{T}=B=-C \text {, i.e., } c_{11}=c_{33}=c_{44}=-c_{13} .
$$

This case reduces to isotropic iff $\mu_{T}=\mu_{L}$ (iff $A=0$ ).

Another example is that of (GWP2) with $\mu_{T}=\mu_{L}$ :

$$
\begin{aligned}
(G \text { WP2iso }) & \left(C+\mu_{L}\right)^{2}=\left(A+\mu_{T}-\mu_{L}\right)\left(B-\mu_{L}\right) \quad \text { and } \quad \mu_{T}=\mu_{L}, \\
\text { i.e., } & 2 \mu_{T}=2 \mu_{L}=-(A+2 C)+\sqrt{A^{2}+4 A(B+C)}, \\
\text { i.e., } & 4 c_{44}=4 c_{66}=-\left(c_{11}+5 c_{12}\right)+\sqrt{\left(c_{11}+c_{12}\right)\left(c_{11}+9 c_{12}+8 c_{33}\right)} .
\end{aligned}
$$

It is clear that this expression for $\mu_{T}$ is real and positive since $A^{2}+4 A(B+C)>(A+2 C)^{2} \geq 0$ by (5). This case reduces to isotropic if and only if $A+\mu_{T}=B$. It follows that two eigenvalues may coincide for all directions of $\xi$, without anisotropy degenerating to isotropy. (See also Chadwick's [53, p39, 44-45] description of this non-isotropic case (GWP2iso) of transverse isotropy with coincident shear modes.)

The slowness surface for GWP. The sheets $1=\lambda(x, s)$ of the slowness surface associated with the Hamiltonian functions $\tau^{2}-\lambda(x, \xi)$ are ellipsoids in the case of GWP (but not generally in the case of (Q1)). That is, $\lambda(x, s)=s^{t} g^{-1}(x) s$ where for $(G W P 1)$ the associated metrics are:

$$
\begin{gathered}
g_{0}^{-1}=\widehat{\mu}_{T} I+\left(\widehat{\mu}_{L}-\widehat{\mu}_{T}\right)(k \otimes k), \quad g_{1}^{-1}=\left(\widehat{A}+\widehat{\mu}_{T}\right) I+\left(\widehat{\mu}_{L}-\widehat{A}-\widehat{\mu}_{T}\right)(k \otimes k), \\
g_{2}^{-1}=\widehat{\mu}_{L} I+\left(\widehat{B}-\widehat{\mu}_{L}\right)(k \otimes k) ;
\end{gathered}
$$

while in case $(G W P 2)$ :

$$
g_{0}^{-1}=\widehat{\mu}_{T} I+\left(\widehat{\mu}_{L}-\widehat{\mu}_{T}\right)(k \otimes k), \quad g_{1}^{-1}=\widehat{\mu}_{L} I, \quad g_{2}^{-1}=\left(\widehat{A}+\widehat{\mu}_{T}\right) I+\left(\widehat{B}-\widehat{A}-\widehat{\mu}_{T}\right)(k \otimes k),
$$

with $\quad \widehat{\mu}_{T}=\mu_{T} / \rho, \quad \widehat{\mu}_{L}=\mu_{L} / \rho, \quad \widehat{A}=A / \rho, \quad \widehat{B}=B / \rho, \quad \widehat{C}=C / \rho$. 
These $g_{0}^{-1}, g_{1}^{-1}, g_{2}^{-1}$ are positive-definite by the strong ellipticity of $\mathbf{C}$. In fact, a matrix of the form $p I+q(k \otimes k)$ has eigenvalues $p, p, p+q$, and so is positive-definite if and only if $p>0$ and $p+q>0$.

In other words, in case (GWP1) the sheets of the slowness surface are given by:

$$
1=\widehat{\mu}_{T}\left|s_{k^{\perp}}\right|^{2}+\widehat{\mu}_{L}\left|s_{k}\right|^{2}, \quad 1=\left(\widehat{A}+\widehat{\mu}_{T}\right)\left|s_{k^{\perp}}\right|^{2}+\widehat{\mu}_{L}\left|s_{k}\right|^{2}, \quad 1=\widehat{\mu}_{L}\left|s_{k^{\perp}}\right|^{2}+\widehat{B}\left|s_{k}\right|^{2},
$$

while in case (GWP2) they are:

$$
1=\widehat{\mu}_{T}\left|s_{k^{\perp}}\right|^{2}+\widehat{\mu}_{L}\left|s_{k}\right|^{2}, \quad 1=\widehat{\mu}_{L}|s|^{2}, \quad 1=\left(\widehat{A}+\widehat{\mu}_{T}\right)\left|s_{k^{\perp}}\right|^{2}+\widehat{B}\left|s_{k}\right|^{2},
$$

where $s_{k^{\perp}}=s-(s \cdot k) k$ and $s_{k}=(s \cdot k) k$.

Conditions for constant multiplicity along rays for transversely isotropic elastodynamics with GWP. In Table 1 we summarize the cases of GWP for which the third mode is disjoint from the others. The vertical axis represents the fiber direction $k$, and the horizontal axis represents any axis orthogonal to $k$. (Slowness surfaces for transverse isotropy have a rotational symmetry around the fiber direction $k$.) The circles in the figures have radius $1 / \sqrt{\mu_{L}}$, and the double circle represents two sheets of the slowness surface that coincide for all $\xi$.

We emphasize that although the disjoint mode is the fastest in three of the four cases (since its wave speed $\sqrt{\lambda(x, \xi)}$ is larger than the others), in fact, the disjoint mode is the slowest in one case for $(C M 1)$, when $B<\mu_{L}<\mu_{T}<A+\mu_{T}$, i.e., $c_{33}<c_{44}<c_{66}<c_{11}$.

\section{The Dynamic Inverse Problem for Anisotropic Elastic Media with a Disjoint Mode}

In this section we prove our main results concerning the dynamic inverse problem for anisotropic elastic media with a disjoint wave mode. Given two elastic media $(\Omega, \rho, \mathbf{C})$ and $(\Omega, \widetilde{\rho}, \widetilde{\mathbf{C}})$, the aim is to describe any relationship between the parameters $\rho$ and $\tilde{\rho}, \mathbf{C}$ and $\tilde{\mathbf{C}}$ that results from the assumption that the surface measurements, in the form of the Dirichlet-to-Neumann maps, agree.

Our primary example is transversely isotropic media that satisfy the strong ellipticity conditions (6) and (CM1) or (CM2) so that the light cone $\tau^{2}=\xi^{t} g_{2}^{-1}(x) \xi$ is always disjoint from the other light cones. (See Table 1.) For these media the Riemannian metrics $g_{i}$, whose geodesic segments are the paths of interior wave propagation, are given by (7) and (8).

We first show that for general anisotropic elastic media with a disjoint wave mode, the Dirichlet-to-Neumann map uniquely determines the travel time through the object for this mode. GWP is not necessary for this result. We assume that there are no trapped rays for the disjoint modes (that is, that it is possible to choose $T$ to be finite).

Theorem 1 (Uniqueness of the travel times of the disjoint mode) Suppose that $\mathbb{R}^{3} \backslash \Omega$ is diffeomorphic to the exterior of a ball in $\mathbb{R}^{3}$. Let $P_{\rho, \mathrm{C}}$ be any system (3) of differential operators for general anisotropic elastodynamics on $\Omega \times[0, T]$ with $\rho>0, \mathbf{C}$ strongly elliptic on $\Omega$, and $\rho, \mathbf{C}$ smooth on $\bar{\Omega}$. Let $\widehat{P}_{\rho, \mathbf{C}}$ be the operator $P_{\rho, \mathbf{C}}$ divided by $\rho$. Writing the determinant of the principal symbol of $\widehat{P}_{\rho, \mathbf{C}}$ as

$$
\operatorname{det} \sigma_{p r}\left(\widehat{P}_{\rho, \mathbf{C}}\right)=-\left(\tau^{2}-\lambda_{0}(x, \xi)\right)\left(\tau^{2}-\lambda_{1}(x, \xi)\right)\left(\tau^{2}-\lambda_{2}(x, \xi)\right),
$$

we suppose that $\lambda_{2}(x, \xi)$ never agrees with $\lambda_{0}(x, \xi)$ or $\lambda_{1}(x, \xi)$ on $T^{*} \Omega \backslash 0$. In addition, let $P_{\widetilde{\rho}, \tilde{\mathbf{c}}}$ be another operator satisfying the same conditions as $P_{\rho, \mathbf{C}}$. Finally, suppose that the parameters $\rho, \widetilde{\rho}$ and $\mathbf{C}, \tilde{\mathbf{C}}$ agree to infinite order at the boundary of $\Omega$, and that the dynamic Dirichlet-to-Neumann maps agree on $\partial \Omega \times(0, T)$ :

$$
\Lambda_{\rho, \mathbf{C}}=\Lambda_{\tilde{\rho}, \tilde{\mathbf{C}}}
$$

where $T$ is larger than the travel time with respect to the disjoint modes between any two boundary points of $\Omega$. Then the travel time along any disjoint-mode bicharacteristic that connects boundary points is determined by the Dirichlet-to-Neumann map. 
Table 1. Cases of GWP with a disjoint slowness surface where $\quad \mathbf{a}=\frac{1}{\sqrt{A+\mu_{T}}}, \quad \mathbf{b}=\frac{1}{\sqrt{B}}, \quad \mathbf{l}=\frac{1}{\sqrt{\mu_{L}}}, \quad \mathbf{t}=\frac{1}{\sqrt{\mu_{T}}}$

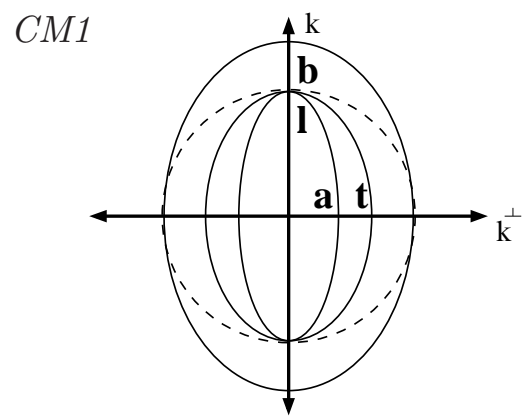

$$
\begin{gathered}
B<\mu_{L}<\mu_{T}<A+\mu_{T} \\
\text { and } \quad C+\mu_{L}=0
\end{gathered}
$$



$$
\begin{gathered}
\mu_{T}<A+\mu_{T}<\mu_{L}<B \\
\text { and } \quad C+\mu_{L}=0
\end{gathered}
$$

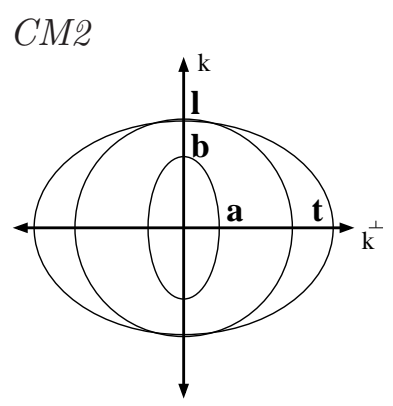

$\mu_{L}>\mu_{T}$



$\mu_{L}=\mu_{T}$

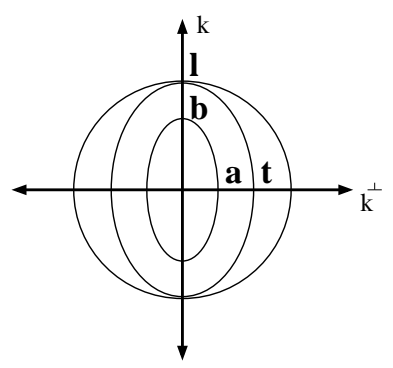

$\mu_{L}<\mu_{T}$

and $\quad \mu_{L}<\min \left\{A+\mu_{T}, B\right\}, \quad\left(C+\mu_{L}\right)^{2}=\left(A+\mu_{T}-\mu_{L}\right)\left(B-\mu_{L}\right)$

The principal difficulty in proving the main result is that in anisotropic elastic media the modes of wave propagation generally correspond to eigenvalues with nonconstant multiplicity. Consequently, characteristics may intersect, and so we cannot conclude that singularities in the elastic waves follow the characteristic curves of the originating mode. The key step is therefore to decouple the system into an equation for the disjoint mode and a reduced system for the other modes. In general, such decoupling can be carried out only in a microlocal sense, that is, by decomposing the symbol of the operator at all orders, especially since we allow variable coefficients in the equation.

We begin by viewing the initial-boundary-value problem (3) in terms of a Cauchy problem in the whole space, for which the description of wave propagation is simplified. Surface measurements in this case are then interpreted as the result of disturbances initiated outside of the object.

\subsection{Extending to $\mathbb{R}^{3}$}

In the Appendix we provide technical details that show that we can extend $\rho, \widetilde{\rho}$ and $\mathbf{C}, \widetilde{\mathbf{C}}$ to be smooth on $\mathbb{R}^{3}$, preserving strong ellipticity, the disjoint mode condition, and, for Corollary 4 GWP of the disjoint mode, and so that $\rho=\widetilde{\rho}$ and $\mathbf{C}=\widetilde{\mathbf{C}}$ in $\mathbb{R}^{3} \backslash \Omega$. We prove this result under the condition that $\Omega$ is diffeomorphic to a ball for general anisotropic elastic media, but this assumption is not needed in the case of transversely isotropic media. 
Next, applying an approach presented in [11] in the case of the wave equation and extended to elastodynamics with residual stress in [15], we compare the solutions $U, \widetilde{U}$, of the initial value problems

$$
\begin{gathered}
\left\{\begin{array}{cl}
\widehat{P}_{\rho, \mathbf{c}} U=0 & \text { in } \mathbb{R}^{3} \times(0, T) \\
\left.U\right|_{t=0}=\psi_{0},\left.\quad\left(\partial_{t} U\right)\right|_{t=0}=\psi_{1} & \text { in } \mathbb{R}^{3}
\end{array}\right. \\
\left\{\begin{array}{cl}
\widehat{P}_{\widetilde{\rho}, \widetilde{\mathbf{C}}} \widetilde{U}=0 & \text { in } \mathbb{R}^{3} \times(0, T) \\
\left.\widetilde{U}\right|_{t=0}=\psi_{0},\left.\quad\left(\partial_{t} \widetilde{U}\right)\right|_{t=0}=\psi_{1} & \text { in } \mathbb{R}^{3}
\end{array}\right.
\end{gathered}
$$

where $\widehat{P}_{\rho, \mathrm{C}}$ is the operator $P_{\rho, \mathrm{C}}$ for elastodynamics, given in (3), divided by the density $\rho$.

We let $\psi_{0} \in H^{s}\left(\mathbb{R}^{3}\right)$ and $\psi_{1} \in H^{s-1}\left(\mathbb{R}^{3}\right), s \geq 0$, be supported on $\mathbb{R}^{3} \backslash \bar{\Omega}$. It follows that if $U$ solves the initial value problem $(10)$ for $(\rho, \mathbf{C})$, then the unique solution of $(10)$ for $(\widetilde{\rho}, \widetilde{\mathbf{C}})$, agrees with $U$ on $\left(\mathbb{R}^{3} \backslash \Omega\right) \times[0, T]$ provided

$$
\Lambda_{\rho, \mathbf{C}}=\Lambda_{\widetilde{\rho}, \widetilde{\mathbf{C}}}
$$

In fact, we show below that $\widetilde{U}$ is the unique weak solution of (10) for $\widehat{P}_{\widetilde{\rho}, \widetilde{\mathbf{c}}}$, with $\widetilde{U}$ defined as

$$
\widetilde{U}= \begin{cases}U & \text { in } \mathbb{R}^{3} \backslash \Omega \\ \widetilde{v} & \text { in } \Omega,\end{cases}
$$

where $\widetilde{v}$ solves the initial-boundary-value problem (2) for $\widehat{P}_{\widetilde{\rho}, \widetilde{\mathbf{C}}}$ with boundary values $\left.\widetilde{v}\right|_{\partial \Omega}=\left.U\right|_{\partial \Omega}$ for $t \in[0, T]$. By construction $U=\widetilde{U}$ on $\left(\mathbb{R}^{3} \backslash \Omega\right) \times[0, T]$.

Since the system for elastodynamics is linear, hyperbolic, and symmetric (see, for example, [7, p. 368] and references therein), there exists a unique weak solution of (10) in $C\left(\mathbb{R}, H^{s}\right) \cap C^{1}\left(\mathbb{R}, H^{s-1}\right), s \geq 0$ [54, page 22]. In particular, for the application below we can take the initial data to be in $H^{s}\left(\mathbb{R}^{3}\right)$ for large enough $s$ so that $U$ and $\nabla U$ are continuous across $\partial \Omega$.

We employ the following weak formulation of the system of equations $\widehat{P}_{\rho, \mathrm{C}} U=0$ on $\mathbb{R}^{3} \times(0, T)$ :

$$
\int_{\mathbb{R}^{3}}\left[\frac{\partial^{2}}{\partial t^{2}} U(x, t)\right] \cdot U(x, t) d x+\int_{\mathbb{R}^{3}} \nabla\left(\rho^{-1} U\right) \cdot C \cdot \nabla U d x=0,
$$

and observe by the divergence theorem that

$$
\begin{aligned}
\int_{\mathbb{R}^{3}} \nabla\left(\widetilde{\rho}^{-1} \widetilde{U}\right) \cdot \widetilde{\mathbf{C}} & \cdot \nabla \widetilde{U} d x=-\int_{\mathbb{R}^{3} \backslash \Omega}\left(\widetilde{\rho}^{-1} U\right) \cdot \nabla \cdot(\widetilde{\mathbf{C}} \cdot \nabla U) d x-\int_{\Omega}\left(\widetilde{\rho}^{-1} \widetilde{v}\right) \cdot \nabla \cdot(\widetilde{\mathbf{C}} \cdot \nabla \widetilde{v}) d x \\
& -\int_{\partial \Omega} \widetilde{\rho}^{-1} U \cdot(\widetilde{\mathbf{C}} \cdot \nabla U) \cdot \nu d x+\int_{\partial \Omega} \widetilde{\rho}^{-1} \widetilde{v} \cdot(\widetilde{\mathbf{C}} \cdot \nabla \widetilde{v}) \cdot \nu d x
\end{aligned}
$$

where $\nu$ is the unit outer normal to $\partial \Omega$. In fact, by (11):

$$
(\widetilde{\mathbf{C}} \cdot \nabla \widetilde{v}) \cdot \nu \underset{\partial \Omega}{\mid}=\Lambda_{\tilde{\rho} \widetilde{\mathbf{C}}}(\widetilde{v} \mid)=\Lambda_{\partial \Omega} \widetilde{\rho}_{\partial \Omega}(U \underset{\partial \Omega}{\mid})=\Lambda_{\rho, \mathbf{C}}(U \underset{\partial \Omega}{\mid})=(\mathbf{C} \cdot \nabla U) \cdot \nu \underset{\partial \Omega}{\mid}=\left.(\widetilde{\mathbf{C}} \cdot \nabla U) \cdot \nu\right|_{\partial \Omega}
$$

since $U$ and $\nabla U$ are continuous across $\partial \Omega$, the parameters agree on the boundary, and $\left.\widetilde{v}\right|_{\partial \Omega}=\left.U\right|_{\partial \Omega}$. We have therefore

$$
\begin{aligned}
& \int_{\mathbb{R}^{3}}\left[\frac{\partial^{2}}{\partial t^{2}} \widetilde{U}\right] \cdot \widetilde{U} d x+\int_{\mathbb{R}^{3}} \nabla\left(\widetilde{\rho}^{-1} \widetilde{U}\right) \cdot \widetilde{\mathbf{C}} \cdot \nabla \widetilde{U} d x \\
& \quad=\int_{\mathbb{R}^{3} \backslash \Omega}\left[\frac{\partial^{2}}{\partial t^{2}} U(x, t)-\rho^{-1} \nabla \cdot(\mathbf{C} \cdot \nabla U)\right] \cdot U(x, t) d x+\int_{\Omega}\left[\frac{\partial^{2}}{\partial t^{2}} \widetilde{v}(x, t)-\widetilde{\rho}^{-1} \nabla \cdot(\widetilde{\mathbf{C}} \cdot \nabla \widetilde{v})\right] \cdot \widetilde{v}(x, t) d x \quad=\quad 0
\end{aligned}
$$

and we conclude that $\widetilde{U}$ is a weak solution on $\mathbb{R}^{3} \times(0, T)$ for $\widehat{P}_{\widetilde{\rho}, \widetilde{\mathbf{C}}}$. 


\subsection{Decoupling the system of differential equations for elastodynamics}

We show in Lemma 2 below that the differential equation governing the third-mode component $v_{2}(x, D) \cdot U$ of the displacement $U$ can be decoupled microlocally at every order from the system of differential equations governing the two other modes, where $v_{2}(x, \xi)$ is the eigenvector of the determinant of the principal symbol associated with the disjoint eigenvalue $\lambda_{2}(x, \xi)$. (See [10] for explicit formulas for $v_{2}$ in the case of transverse isotropy with GWP.) In particular, we modify $v_{2}(x, D) \cdot U$ with asymptotically lower order terms to arrive at a solution $W^{3}$ of the initial value problem (16) for a scalar, strictly hyperbolic equation. We conclude in Step 2 below that the singularities of $W^{3}$ may be described in terms of those of the initial data for the Cauchy problem (10) via propagation of singularities results for scalar, strictly hyperbolic equations. We will choose initial data for (10) so that the displacement $U$ has wave front set that lies only in the light cone for the third wave mode.

The proof of Lemma 2 extends a result of Taylor [44] (see also [55]), but there are two key differences between our approach and that of Taylor. First, we decouple the operator over a relatively long period $[0, T]$ of time (enough for the third mode disturbance from a point $x_{0} \notin \bar{\Omega}$ to travel through, and exit, the object). Second, we do not assume that the eigenvalues of the principal symbol are all distinct; nor is the system of real principal type.

Here, we adapt the approach taken by Stolk and de Hoop, who treat seismic inverse scattering in anisotropic media [3], under the assumption that the multiplicity of each decoupled mode is constant, at least microlocally. As in [3], we cannot necessarily use the eigenvectors of the principal symbol to accomplish the decoupling. In fact, in the case of transversely isotropic elastodynamics with GWP only one of the eigenvectors $v_{2}(x, \xi)$ of the principal symbol is smooth on the entire cotangent bundle. Furthermore, in case $(G W P$ 2$)$ for fixed $x \in \Omega$ and any $\widehat{\xi}=\xi /|\xi|$ we have that $v_{2}(x, \widehat{\xi})$ can be roughly parallel to $\widehat{\xi}$, and so there is no smooth vector field $e(x, \widehat{\xi})$ orthogonal to $v_{2}(x, \widehat{\xi})$ on the unit sphere bundle. For this reason we decouple the system only locally in the cotangent bundle. Stolk and de Hoop also decouple the system microlocally, but in a neighborhood of a given (and arbitrary) point and direction, while we decouple the system in a neighborhood of a bicharacteristic for $t \in[0, T]$, $T$ not necessarily small.

Informally, we choose initial data for $U$ with "minimal" singularity, in the sense that the initial data lack smoothness at only one point $x_{0} \in \mathbb{R}^{3} \backslash \Omega$ and along only one direction $\xi_{0}$. Next, we partially diagonalize the principal symbol of the spatial part of $\widehat{P}_{\rho, \mathbf{C}}$ in a conic neighborhood of the disjoint mode bicharacteristics through $\left(x_{0}, \xi_{0}\right)$ by explicitly constructing a family of invertible matrices $Q^{0}(x, t, \xi)$ that are smooth on the entire space $\left(T^{*} \mathbb{R}^{3}\right) \times[0, T]$. We then apply Taylor's construction to obtain a partial diagonalization of the full symbol. For this we need only assume that $\lambda_{2}(x, \xi)$ is disjoint. (It follows that the associated eigenvector $v_{2}(x, \xi)$ of the principal symbol is smooth on $T^{*} \mathbb{R}^{3}$.) In this fashion we guarantee that only the disjoint wave mode carries the singularities of $U$.

Below, we use Hörmander notation for pseudo-differential operators; that is, we denote by $O P S_{\rho, \delta}^{m}$ the class of pseudo-differential operator with symbols in $S_{\rho, \delta}^{m}, 0 \leq \rho, \delta \leq 1$, by $O P S_{\mathrm{cl}}^{m}$ the class of operators with polyhomogeneous symbols of degree $m$, and by $O P S^{-\infty}$ the class of smoothing operators.

\section{Step 1 (Decoupling the disjoint mode of $\widehat{P}_{\rho, \mathbf{C}}$ )}

We denote by $\sigma_{x}(A)$ the reduced symbol on $T^{*} \mathbb{R}^{3}$ of a pseudo-differential operator $A\left(x, t, D, D_{t}\right)$, and by $C(t)$ the canonical relation induced by the Hamilton vector field $H_{\lambda_{2}(x, \xi)}$ on the cotangent bundle $T^{*} \mathbb{R}^{3}$.

Lemma 2 (Decoupling the system for elastodynamics) Let $P_{\rho, \mathrm{c}}$ be a system satisfying the conditions of Theorem 1 , but on all of $\mathbb{R}^{3}$ and with $\rho, \mathbf{C}$ constant outside a compact set in $\mathbb{R}^{3}$. Then for any fixed $\left(x_{0}\right.$, $\left.\xi_{0}\right)$ in $T^{*} \mathbb{R}^{3} \backslash 0$ there exists an open, conic neighborhood $\mathfrak{U}$ of $\left(x_{0}, \xi_{0}\right)$ and a microlocally invertible, pseudo-differential operator $Q(x, t, D) \in O P S_{1,0}^{0}\left(\mathbb{R}^{3}\right)$, depending smoothly on $t \in[0, T]$, such that:

$$
\sigma_{x}\left(Q^{-1} \widehat{P}_{\rho, \mathbf{C}} Q(t, x, D)\right)=\sigma_{x}\left(\begin{array}{cc}
I & \partial_{t}^{2}
\end{array}-\left(\begin{array}{cc}
A_{01}(x, t, D) & 0 \\
0 & a_{2}(x, t, D)
\end{array}\right)+R\right)
$$

in $C(t) \mathfrak{U}, t \in[0, T]$, where $I$ is the identity, $R \in O P S^{-\infty}$ is a smoothing operator, $A_{01}(x, t, D)$ is a $2 \times 2$ symmetric, matrix-valued operator in $O P S_{1,0}^{2}\left(\mathbb{R}^{3}\right)$, and $a_{2}(x, t, D)$ is a scalar operator in OPS$S_{1,0}^{2}\left(\mathbb{R}^{3}\right)$ with principal symbol:

$$
a_{2}^{0}(x, \xi)=\lambda_{2}(x, \xi)
$$


Proof. The time-dependent part of $\widehat{P}_{\rho, \mathrm{C}}$ is already in diagonal form, so we need only decouple the elliptic part in $C(t) \mathfrak{U}, t \in[0, T]$, where $\mathfrak{U}$, to be chosen later, is a conic neighborhood of $\left(x_{0}, \xi_{0}\right)$ in $T^{*} \mathbb{R}^{3}$. The first step is to partially diagonalize the principal symbol of the elliptic part $\rho^{-1}(x) C^{i j k l}(x) \xi_{j} \xi_{l}$ of $\widehat{P}_{\rho, \mathbf{C}}$ in $C(t) \mathfrak{U}, t \in[0, T]$, in a smooth fashion. We then extend the resulting principal symbol smoothly to $\mathbb{R}^{3} \times[0, T]$.

We begin by constructing a smooth, orthogonal matrix $Q^{0}(x, t, \xi)=\left[e_{0}(x, t, \xi), e_{1}(x, t, \xi), e_{2}(x, t, \xi)\right]$ on $T^{*}\left(\mathbb{R}^{3} \times[0, T]\right)$, homogeneous of degree 0 in $\xi$, so that

$$
\left(Q^{0}\right)^{-1}(x, t, \xi) \rho^{-1}(x) C^{i j k l}(x) \xi_{j} \xi_{l} Q^{0}(x, t, \xi)=\left(\begin{array}{cc}
A_{01}^{0}(x, t, \xi) & 0 \\
0 & a_{2}^{0}(x, \xi)
\end{array}\right) \quad \text { in } C(t) \mathfrak{U}
$$

with $a_{2}^{0}(x, \xi)=\lambda_{2}(x, \xi)$ and $A_{01}^{0}(x, t, \xi)$ a $2 \times 2$ symmetric, matrix-valued function. The eigenvalues of $A_{01}^{0}$ are by construction the first two eigenvalues $\lambda_{0}(x, \xi)$ and $\lambda_{1}(x, \xi)$ of $\rho^{-1} \mathrm{C}^{i j k l} \xi_{j} \xi_{l}$ on $C(t) \mathfrak{U}$. It follows that $A_{01}^{0}$ and $a_{2}^{0}$ have disjoint spectra. (We remark that for such a $Q^{0}$ both $A_{01}^{0}(x, t, \xi)$ and $a_{2}^{0}(x, \xi)$ are homogeneous of degree two in $\xi$ away from $\xi=0$.)

It is not necessarily possible to choose $e_{0}, e_{1}, e_{2}$ to agree with the eigenvectors of the principal symbol of $\widehat{P}_{\rho, \mathrm{C}}$ on all of $T^{*} \mathbb{R}^{3}$. In fact, in the case of transverse isotropy with GWP the eigenvectors $v_{0}, v_{1}$ are not smooth at $\xi \in \operatorname{Span}\{k\}$, as is shown in [10]. In addition, in case (GWP2) for any $x \in \Omega$ and any $\widehat{\xi}=\xi /|\xi|$ we have that $v_{2}(x, \widehat{\xi})$ can be roughly parallel to $\widehat{\xi}$, in which case there is no smooth vector field $e(x, \widehat{\xi})$ orthogonal to $v_{2}(x, \widehat{\xi})$ on the unit sphere bundle.

To choose $e_{0}, e_{1}, e_{2}$ we define $\widehat{\mathfrak{U}}$ to be the product $B_{x_{0}}(\epsilon) \times s_{\xi_{0}}$ in the unit co-sphere bundle $S^{*} \mathbb{R}^{3}=\{(x, \widehat{\xi})$ : $|\widehat{\xi}|=1\}$, where $B_{x_{0}}(\epsilon)$ is the ball in $\mathbb{R}^{3}$ about $x_{0}$ of radius $\epsilon$, and $s_{\xi_{0}}=\left\{\widehat{\xi}\left|\widehat{\xi} \cdot \xi_{0} /\right| \xi_{0} \mid>1-\epsilon\right\}$ consists of the unit sphere vectors with scalar projection onto $\xi_{0} /\left|\xi_{0}\right|$ greater than $1-\epsilon$. Here we take $\epsilon>0$ small enough so that the third eigenvector $v_{2}(x, \widehat{\xi})$ of the principal symbol $\widehat{P}_{\rho, \mathbf{C}}^{0}$ is almost constant within $\widehat{\mathfrak{U}}$. We define $e_{2}(x, 0, \widehat{\xi})$ to agree with $v_{2}(x, \widehat{\xi})$ in $\widehat{\mathfrak{U}}$, and for each $x \in B_{x_{0}}(\epsilon)$ we extend $e_{2}(x, 0, \widehat{\xi})$ smoothly to $S_{x}^{*} \mathbb{R}^{3}$ so that $e_{2}(x, 0, \widehat{\xi})=v_{2}\left(x_{0}, \widehat{\xi}_{0}\right)$ is constant for $\widehat{\xi}$ bounded away from $s_{\xi_{0}}$. We then extend $e_{2}(x, 0, \widehat{\xi})$ smoothly to $S^{*} \mathbb{R}^{3}$ so that $e_{2}(x, 0, \widehat{\xi})=v_{2}\left(x_{0}, \widehat{\xi}_{0}\right)$ is constant on the complement of $\widehat{\mathfrak{U}}$ in $S^{*} \mathbb{R}^{3}$. It follows that for some smooth $e_{0}, e_{1}(x, 0, \widehat{\xi}), Q^{0}$ is orthogonal and smooth on $S^{*} \mathbb{R}^{3}$. We define $\mathfrak{U}$ to be the conic extension $\{(x, \xi)|(x, \widehat{\xi}) \in \widehat{\mathfrak{U}}, \widehat{\xi}=\xi /| \xi \mid\}$ in $\left(T^{*} \mathbb{R}^{3}\right) \backslash 0$ of $\widehat{\mathfrak{U}}$. We extend $Q^{0}(x, 0, \widehat{\xi})$ to $T^{*} \mathbb{R}^{3} \backslash 0$ so that $Q^{0}$ is homogeneous of order zero in $\xi$, and then we extend $Q^{0}$ smoothly on the flow-out $C(t)\left(T^{*} \mathbb{R}^{3} \backslash 0\right), t \in[0, T]$, to be constant along the bicharacteristics. We denote by $Q^{0}(x, t, D)$ the family of pseudo-differential operators on $\mathbb{R}^{3}$ parametrized by $t \in[0, T]$ and with symbol $Q^{0}(x, t, \xi)$. $Q^{0}(x, t, D)$ is microlocally invertible on $\left(T^{*} \mathbb{R}^{3}\right) \backslash 0$. It follows that

$$
\begin{aligned}
& \left(Q^{0}\right)^{-1}(x, t, \xi) \sigma_{x}\left(\widehat{P}_{\rho, \mathbf{C}}\right) Q^{0}(x, t, \xi) \\
& \quad=\mathrm{I} \partial_{t}^{2}-\left(\begin{array}{cc}
A_{01}^{0}(x, t, \xi) & 0 \\
0 & a_{2}^{0}(x, \xi)
\end{array}\right)+B(x, t, \xi) \quad \text { in } C(t) \mathfrak{U}, \quad t \in[0, T],
\end{aligned}
$$

where $B(x, t, \xi) \in S_{\mathrm{cl}}^{1}$, and $A_{01}^{0}(x, t, \xi), a_{2}^{0}(x, \xi) \in S_{\mathrm{cl}}^{2}$ are symbols in $C(t) \mathfrak{U}, t \in[0, T]$, depending smoothly on $t$. Above, $\sigma_{x}(P)$ denotes the reduced symbol in $T^{*} \mathbb{R}^{3}$ of an operator $P\left(x, t, D, D_{t}\right)$ with respect to $x$.

We next extend $A_{01}^{0}(x, t, \xi)$ and $a_{2}^{0}(x, \xi)$ smoothly to $\left(T^{*} \mathbb{R}^{3}\right) \times[0, T]$ so that the right side of (13) defines a pseudo-differential operator on $\mathbb{R}^{3}$, depending smoothly on $t$, that agrees with the diagonalization of the principal symbol of $\widehat{P}_{\rho, \mathrm{C}}$ in $C(t) \mathfrak{U}$. In fact, since the eigenvalues $\lambda_{i}(x, \xi), i=0,1,2$, of the principal symbol $\rho^{-1}(x) C^{i j k l}(x) \xi_{j} \xi_{l}$ of the elliptic part of $\widehat{P}_{\rho, \mathbf{C}}$ are smooth on the cotangent bundle $T^{*} \mathbb{R}^{3}$, we can extend $a_{2}^{0}$ to agree with $\lambda_{2}(x, \xi)$ on $T^{*} \mathbb{R}^{3}$. In addition, below we extend $A_{01}^{0}$ to $T^{*} \mathbb{R}^{3}$ at $t=0$; it follows that we can extend $A_{01}^{0}$ to $\left(T^{*} \mathbb{R}^{3}\right) \times[0, T]$ to be constant along bicharacteristics. To extend $A_{01}^{0}$ to $T^{*} \mathbb{R}^{3}$ at $t=0$ we observe that 
$v_{2}(x, \xi), \quad \rho^{-1}(x) C^{i j k l}(x) \xi_{j} \xi_{l}=\sum_{i=0,1,2} \lambda_{i}\left(v_{i} \otimes v_{i}\right)$ are smooth on $T^{*} \mathbb{R}^{3}$, so

$$
\begin{aligned}
A_{01}^{0}(x, 0, \xi) & =\left(\begin{array}{c}
e_{0} \\
e_{1}
\end{array}\right)\left(\begin{array}{ll}
v_{0} & v_{1}
\end{array}\right)\left(\begin{array}{cc}
\lambda_{0} & 0 \\
0 & \lambda_{1}
\end{array}\right)\left(\begin{array}{l}
v_{0} \\
v_{1}
\end{array}\right)\left(\begin{array}{ll}
e_{0} & e_{1}
\end{array}\right) \\
& =\left(\begin{array}{c}
e_{0} \\
e_{1}
\end{array}\right) \rho^{-1}(x) \mathbf{C}(x)[\cdot, \xi, \cdot, \xi]\left(e_{0} e_{1}\right)-\left(\begin{array}{l}
e_{0} \\
e_{1}
\end{array}\right)\left[\lambda_{2} v_{2} \otimes v_{2}\right]\left(\begin{array}{ll}
e_{0} & e_{1}
\end{array}\right)
\end{aligned}
$$

is smooth and has eigenvalues $\lambda_{0}, \lambda_{1}$ on $T^{*} \mathbb{R}^{3}$.

We decouple the resulting pseudo-differential system

$$
\left[\mathrm{I} \partial_{t}^{2}-\left(\begin{array}{cc}
A_{01}^{0}(x, t, D) & 0 \\
0 & a_{2}^{0}(x, D)
\end{array}\right)+B(x, t, D)\right] w=0 \quad \text { on } \quad\left(T^{*} \mathbb{R}^{3}\right) \times[0, T]
$$

at every order as in [44]. In fact, we construct an operator $K=K(t, x, D) \in O P S_{\mathrm{cl}}^{-1}\left(\mathbb{R}^{3}\right)$, depending smoothly on $t$, with which we may transform this system, via

$$
W=(\mathrm{I}+K) w
$$

to one that is decoupled at every order:

$$
\left[\mathrm{I} \partial_{t}^{2}-\left(\begin{array}{cc}
A_{01}(x, t, D) & 0 \\
0 & a_{2}(t, x, D)
\end{array}\right)+R\right] W \in C^{\infty},
$$

with $A_{01} \in O P S_{\mathrm{cl}}^{2}\left(\mathbb{R}^{3}\right)$ a $2 \times 2$ matrix, $a_{2} \in O P S_{\mathrm{cl}}^{2}\left(\mathbb{R}^{3}\right)$ scalar, having principal parts given by $A_{01}^{0}(x, t, D)$ and $a_{2}^{0}(x, D)$, respectively, and depending smoothly on $t$.

\section{Step 2 (Constructing a solution $W$ of the decoupled system with wave front set occurring only along certain bicharacteristics of the disjoint mode).}

Our next step consists in constructing a solution of the decoupled system with singularities only in the third component and along prescribed directions. We use the notion of wave front set of a distribution, which describes the points and directions at which the distribution differs from a smooth function. It is possible to construct distributions with wave front set consisting of a single point and a single direction. We refer to [56], [57, Chapter 5, Example 1.2] for the precise definition and examples.

Let $\left(x_{0}, \xi_{0}\right)$ be any point in $T^{*} \mathbb{R}^{3} \backslash 0$ with $x_{0} \notin \bar{\Omega}$, and let $A_{01} \in O P S_{\mathrm{cl}}^{2}\left(\mathbb{R}^{3}\right)$ be any $2 \times 2$ symmetric system, with principal part $A_{01}^{0}(x, t, D)$, depending smoothly on $t$. Let $a_{2}(x, t, D) \in O P S_{\mathrm{cl}}^{2}\left(\mathbb{R}^{3}\right)$ be scalar, depending smoothly on $t$, with principal part $a_{2}^{0}(x, D)$, having symbol $a_{2}^{0}(x, \xi)=\lambda_{2}(x, \xi)$. We consider the following initial-value problem on $\left(T^{*} \mathbb{R}^{3}\right) \times[0, T]$ :

$$
\left\{\begin{array}{l}
{\left[\mathrm{I} \partial_{t}^{2}-\left(\begin{array}{cc}
A_{01}(x, t, D) & 0 \\
0 & a_{2}(t, x, D)
\end{array}\right)\right] W=0} \\
\left.W\right|_{t=0}=W_{0} \quad \text { and }\left.\quad\left(\partial_{t} W\right)\right|_{t=0}=W_{1} .
\end{array}\right.
$$

We will establish that, for regular enough initial data $W_{0}, W_{1}$, there exist solutions $W$ such that

where

$$
\mathrm{WF}(W)=\Gamma^{+} \cup \Gamma^{-} \text {, }
$$

$$
\Gamma^{ \pm}=\left\{(x, t, \xi, \tau) \mid \tau=\mp \sqrt{\lambda_{2}(x, \xi)}, \quad(x(0), \xi(0))=\left(x_{0}, \xi_{0}\right)\right\}
$$

are the forward and backward null bicharacteristics for $\tau^{2}-\lambda_{2}(x, \xi)$ that pass over $\left(x_{0}, \xi_{0}\right) \in T^{*} \mathbb{R}^{3} \backslash 0$. 
We begin by observing that the first two components $W^{i}, i=1,2$, solve the initial-value problem

$$
\left\{\begin{array}{l}
{\left[\mathrm{I} \partial_{t}^{2}-A_{01}(x, t, D)\right]\left(\begin{array}{l}
W^{1} \\
W^{2}
\end{array}\right)=0} \\
\left.W^{i}\right|_{t=0}=W_{0}^{i} \quad \text { and }\left.\quad\left(\partial_{t} W^{i}\right)\right|_{t=0}=W_{1}^{i}, \quad i=1,2,
\end{array}\right.
$$

which is well posed. In fact, since $A_{01}^{0}$ is symmetric, $K$ is also symmetric by $\left[49,(1.5)\right.$ and (1.6), page 196], and so $A_{01}$ is symmetric at every order. Therefore, I $\partial_{t}^{2}-A_{01}(x, t, D)$ can be transformed into a first-order, symmetric hyperbolic system. We first observe that, since $A_{01}^{0}$ is nonpositive-definite, there exists an operator $\sqrt{-A_{01}^{0}} \in O P S_{\mathrm{cl}}^{1}$ such that $-A_{01}^{0}=\left(\sqrt{-A_{01}^{0}}\right)^{2} \bmod O P S_{\mathrm{cl}}^{1}$. Moreover, $A_{01}^{0}$ is elliptic, so that a (two-sided) parametrix $B=B(x, t, D) \in O P S_{\mathrm{cl}}^{-1}$ exists for $\sqrt{-A_{01}^{0}}$. If we set

$$
y_{1}=\sqrt{-A_{01}^{0}}\left(\begin{array}{l}
W^{1} \\
W^{2}
\end{array}\right), \quad y_{2}=\partial_{t}\left(\begin{array}{c}
W^{1} \\
W^{2}
\end{array}\right),
$$

then the original system for $W_{1}$ and $W_{2}$ is equivalent to the following $4 \times 4$ first-order system for $y_{1}$ and $y_{2}$ modulo smoothing:

$$
\left(\begin{array}{cc}
\mathrm{I} \partial_{t} & 0 \\
0 & \mathrm{I} \partial_{t}
\end{array}\right)-\left(\begin{array}{cc}
0 & -\sqrt{-A_{01}^{0}} \\
\sqrt{-A_{01}^{0}} & 0
\end{array}\right)+\left(\begin{array}{cc}
0 & \left(-\partial_{t} \sqrt{-A_{01}^{0}}\right) B \\
0 & L B
\end{array}\right)\left(\begin{array}{l}
y_{1} \\
y_{2}
\end{array}\right)=0
$$

where $L(x, t, D) \in O P S_{\mathrm{cl}}^{1}$. This system is symmetric hyperbolic (as by construction $\sqrt{-A_{01}^{0}}{ }^{*}=\sqrt{-A_{01}^{0}} \bmod O P S_{\mathrm{cl}}^{0}$ ) and hence well-posed. (See, for example, Theorem 2.3, page 75, in [49].)

In addition, the third component $W^{3}$ solves the initial-value problem

$$
\left\{\begin{array}{c}
\left(\partial_{t}^{2}-a_{2}(x, t, D)\right) W^{3}=\left(\partial_{t}^{2}-\lambda_{2}(x, D)+L(x, t, D)\right) W^{3}=0 \\
\left.W^{3}\right|_{t=0}=W_{0}^{3} \quad \text { and }\left.\quad\left(\partial_{t} W^{3}\right)\right|_{t=0}=W_{1}^{3}
\end{array}\right.
$$

This problem is also well posed since the scalar pseudo-differential operator $\partial_{t}^{2}-\lambda_{2}(x, D)+L(x, t, D)$ is strictly hyperbolic due to the fact that the principal symbol $\tau^{2}-\lambda_{2}(x, \xi)$ has distinct, real $\operatorname{roots} \tau= \pm \sqrt{\lambda_{2}(x, \xi)}$. For media with GWP (16) is a wave equation with respect to the metric $g_{2}$, up to lower-order terms.

We now choose special initial data $W_{0}$ and $W_{1}$ for the initial-value problem (15), following [13], [15], so that $\mathrm{WF}\left(W_{i}\right), i=0,1$, is supported at a single point, is directed along a single ray, and occurs only in the third component. Specifically, for $x_{0} \notin \bar{\Omega}$ and $\xi_{0} \neq 0$, we set

$$
W_{0}(x)=h(x)\left(\begin{array}{l}
0 \\
0 \\
1
\end{array}\right) \quad \text { and } \quad W_{1}=0,
$$

where $h(x)$ is a scalar function in $C^{m}\left(\mathbb{R}^{3}\right)$ (for some arbitrarily large, but finite, $m$ ) with wave front set $\left\{\left(x_{0}, \alpha \xi_{0}\right)\right.$ : $\alpha>0\}$. Distributions with wave front set on a ray are constructed in [56], [58], and [57, Chapter 5, Example 1.2].

It follows that $\mathrm{WF}\left(\left.W^{i}\right|_{t=0}\right)=\emptyset, i=1,2$, so by the well-posedness of the decoupled system (14) $W^{1}(x, t)$ and $W^{2}(x, t)$ are smooth on $\mathbb{R}^{3} \times[0, T)$. That is,

$$
\mathrm{WF} W=\mathrm{WF} W^{3} \text {. }
$$

Also, since $W^{3}$ solves the scalar equation (16) on $\mathbb{R}^{3} \times(0, T), \mathrm{WF}\left(W^{3}\right)$ is given as the flow-out of $\mathrm{WF}\left(\left.W^{3}\right|_{t=0}\right)=$ $\left\{\left(x_{0}, \alpha \xi_{0}\right): \alpha>0\right\}$ in the characteristic set along the Hamilton vector field $H_{\tau^{2}-\lambda_{2}(x, \xi)}$. This result is a consequence of Egorov's Theorem, observing that the principal symbol is time-independent. (See, for example, [49, Chapter VIII, Theorem 1.2].) We conclude that

$$
\mathrm{WF}(W)=\mathrm{WF}\left(W^{3}\right)=C(t)\left(x_{0}, \xi_{0}\right)=\Gamma^{+} \cup \Gamma^{-} .
$$




\section{Step 3 (Constructing displacements $U$ with wave front set occurring only along certain bicharacter- istics of the disjoint mode).}

In this last step, we show how we can construct solutions $U$ of the coupled system (10) in such a way that singularities occur only along the prescribed bicharacteristics $\Gamma^{+}$and $\Gamma^{-}$of the disjoint wave mode. In fact, we show that we can represent $U$ as $Q(x, t, D) \psi(x, t, D) W$ plus a smooth remainder, where $Q$ diagonalizes the full symbol as in Step 1, $W$ is a solution of the decoupled system obtained in Step 2, and $\psi(x, t, \xi)$ is a cutoff function supported in the conic neighborhood $C(t) \mathfrak{U}$. Therefore, there is no contribution to the wave-front set of the solution $U$ outside of $C(t) \mathfrak{U}$.

Let $P_{\rho, \mathrm{C}}$ be any system (3) of differential operators for general anisotropic elastodynamics satisfying the conditions of Lemma 2 , and let $\left(x_{0}, \xi_{0}\right)$ be any point in $T^{*} \mathbb{R}^{3} \backslash 0$ with $x_{0} \notin \bar{\Omega}$. Then there exist solutions $U$ of (10) with initial values $\left.U\right|_{t=0},\left.\left(\partial_{t} U\right)\right|_{t=0}$ supported outside $\Omega$, and with

$$
\mathrm{WF}(U)=\Gamma^{+} \cup \Gamma^{-},
$$

where $\Gamma^{+}$and $\Gamma^{-}$are given in Step 2.

We start by choosing $\psi(x, t, \xi)$ to be a smooth cut-off function supported in the conic neighborhood $C(t) \mathfrak{U}$ that appears in the decoupling of the operator for elastodynamics in Step 1. In particular, suppose that $\psi$ is homogeneous of order 0 , constant along bicharacteristics, and identically one on a conic neighborhood $C(t) \mathfrak{U}_{1}$ of $\left(x_{0}, \xi_{0}\right)$ with $\mathfrak{U}_{1} \subset \mathfrak{U}$. Let $\psi(x, t, D) \in O P S_{\mathrm{cl}}^{0}\left(\mathbb{R}^{3}\right)$ be the associated pseudo-differential operator on $\mathbb{R}^{3}$, depending smoothly on the parameter $t$. Let

$$
\bar{U}=Q(x, t, D) \psi(x, t, D) W
$$

where $Q(x, t, D)=Q^{0}(x, t, D)(I+K)^{-1}$, with $K=K(t, x, D) \in O P S_{\mathrm{cl}}^{-1}\left(\mathbb{R}^{3}\right)$ defined in the decoupling in Step 1 . Then $\mathrm{WF}(\bar{U})=\mathrm{WF}(W)=\Gamma^{+} \cup \Gamma^{-}$since $Q$ has principal symbol which is invertible (and so $Q$ is elliptic), and since $\psi(x, t, \xi) \equiv 1$ on a neighborhood of $\mathrm{WF}(W)$. We next conclude that if $U$ solves (10), and $U-\bar{U}$ has smooth initial values, then $U-\bar{U}$ is smooth by the $C^{\infty}$ well-posedness of $P_{\rho, \mathbf{C}}$, so that

$$
\mathrm{WF}(U)=\mathrm{WF}(\bar{U})=\Gamma^{+} \cup \Gamma^{-} .
$$

In fact, we show below that $\widehat{P}_{\rho, \mathbf{C}} \bar{U} \in \mathbf{C}^{\infty}$. We write $C(t) \mathfrak{U}(x)=\left\{\xi \in \mathbb{R}^{3} \mid x=x(t), \xi=\xi(t),(x(t), \xi(t)) \in C(t) \mathfrak{U}\right\}$ so that

$$
\begin{aligned}
& Q^{-1}(x, t, D) \widehat{P}_{\rho, \mathbf{C}} \bar{U}=\left[Q^{-1} \widehat{P}_{\rho, \mathbf{C}} Q(x, t, D)\right]\left[Q^{-1}(x, t, D) \bar{U}\right] \\
& =\int_{\mathbb{R}^{3}} e^{i x \cdot \xi} \sigma_{x}\left(Q^{-1} \widehat{P}_{\rho, \mathbf{C}} Q(x, t, D)\right) \mathcal{F}_{x}\left[Q^{-1}(x, t, D) \bar{U}\right] d \xi \\
& =\int_{C(t) \mathfrak{U}(x)} e^{i x \cdot \xi} \sigma_{x}\left(Q^{-1} \widehat{P}_{\rho, \mathbf{C}} Q(x, t, D)\right) \mathcal{F}_{x}\left[Q^{-1}(x, t, D) \bar{U}\right] d \xi \\
& +\int_{\mathbb{R}^{3} \backslash C(t) \mathfrak{U}(x)} e^{i x \cdot \xi} \sigma_{x}\left(Q^{-1} \widehat{P}_{\rho, \mathbf{C}} Q(x, t, D)\right) \mathcal{F}_{x}\left[Q^{-1}(x, t, D) \bar{U}\right] d \xi \\
& =\int_{\mathbb{R}^{3}} e^{i x \cdot \xi} \sigma_{x}\left[\mathrm{I} \quad \partial_{t}^{2}-\left(\begin{array}{cc}
A_{01}(x, t, D) & 0 \\
0 & a_{2}(x, t, D)
\end{array}\right)+R\right] \cdot \mathcal{F}_{x}(\psi(x, t, D) W) d \xi \\
& -\int_{\mathbb{R}^{3} \backslash C(t) \mathfrak{U}(x)} e^{i x \cdot \xi} \sigma_{x}\left[\mathrm{I} \quad \partial_{t}^{2}-\left(\begin{array}{cc}
A_{01}(x, t, D) & 0 \\
0 & a_{2}(x, t, D)
\end{array}\right)+R\right] \cdot \mathcal{F}_{x}(\psi W) d \xi \\
& +\int_{\mathbb{R}^{3} \backslash C(t) \mathfrak{U}(x)} e^{i x \cdot \xi} \sigma_{x}\left(Q^{-1} \widehat{P}_{\rho, \mathbf{C}}\right) \mathcal{F}_{x} \bar{U} d \xi
\end{aligned}
$$




$$
\begin{aligned}
& =\left[\mathrm{I} \quad \partial_{t}^{2}-\left(\begin{array}{cc}
A_{01}(x, t, D) & 0 \\
0 & a_{2}(x, t, D)
\end{array}\right)\right] W+F^{\prime} \\
& -\left[\mathrm{I} \quad \partial_{t}^{2}-\left(\begin{array}{cc}
A_{01}(x, t, D) & 0 \\
0 & a_{2}(x, t, D)
\end{array}\right)\right][1-\psi(x, t, D)] W=F^{\prime \prime},
\end{aligned}
$$

where $R$ is smoothing, and $F^{\prime}, F^{\prime \prime}$ are smooth since $W$ solves $(15),[1-\psi(x, t, D)] W$ is smooth on $\mathbb{R}^{3}$, and since $\bar{U}$ and $W$ are smooth outside $C(t) \mathfrak{U}$.

\subsection{Uniqueness of travel times between boundary points}

Here we complete the proof of Theorem 1, and apply it to the case of transverse isotropy with (CM1) or (CM2). A consequence of the decoupling of the system for elastodynamics is that the Dirichlet-to-Neumann map uniquely determines the metric $g_{2}$ up to pull-back by a diffeomorphism fixing the boundary (under certain conditions on $\Omega$ and $g_{2}$ ). We reduce the problem to the well known boundary rigidity problem for Riemannian manifolds, which is to show that the boundary distance function, the geodesic distance function between any two boundary points, uniquely determines the metric. (See, e.g., [29].)

Proof of Theorem 1. Let $(\Omega, \rho, \mathbf{C})$ and $(\Omega, \widetilde{\rho}, \widetilde{\mathbf{C}})$ be two elastic media satisfying the hypotheses of the theorem. Then, as in Section 3.1, the material parameters can be extended smoothly to $\mathbb{R}^{3}$ so that $\rho=\widetilde{\rho}$ and $\mathbf{C}=\widetilde{\mathbf{C}}$ outside $\Omega$. It follows that $Q(x, t, \xi)$ and $\widetilde{Q}(x, t, \xi)$, and $\psi(x, t, \xi)$ and $\widetilde{\psi}(x, t, \xi)$, may be defined to be the same (respectively) for small $t$ and $x \notin \Omega$. Here $Q(x, t, \xi)$ is the decoupling operator of Step 1 and $\psi(x, t, \xi)$ is the pseudodifferential cut-off of Step 3. We conclude that $\sigma_{x}(Q \psi(x, 0, D))=\sigma_{x}(\widetilde{Q} \widetilde{\psi}(x, 0, D))$ and $\sigma_{x}\left(\partial_{t}(Q \psi)(x, 0, D)\right)=\sigma_{x}\left(\partial_{t}(\widetilde{Q} \widetilde{\psi})(x, 0, D)\right)$ since $\psi(x, t, \xi)$ is supported away from $\Omega$ for small $t$. In Step 2 we construct approximate displacements $\bar{U}=Q \psi W, \widetilde{\bar{U}}=\widetilde{Q} \widetilde{\psi} \widetilde{W}$ on $\mathbb{R}^{3} \times[0, T]$ for $\rho, \mathbf{C}$ and $\widetilde{\rho}, \widetilde{\mathbf{C}}$, respectively, with $\mathrm{WF}(\bar{U})=\Gamma^{+} \cup \Gamma^{-}$and $\mathrm{WF}(\widetilde{\bar{U}})=\widetilde{\Gamma}^{+} \cup \widetilde{\Gamma}^{-}$. It follows that $\left.\bar{U}\right|_{t=0}=(Q \psi)(x, 0, D) W_{0}=(\widetilde{Q} \widetilde{\psi})(x, 0, D) W_{0}=\left.\widetilde{\bar{U}}\right|_{t=0}$. Also, $\left.\left(\partial_{t} \bar{U}\right)\right|_{t=0}=\partial_{t}(Q \psi)(x, 0, D) W_{0}+(Q \psi)(x, 0, D) W_{1}=\partial_{t}(\widetilde{Q} \widetilde{\psi})(x, 0, D) W_{0}+(\widetilde{Q} \widetilde{\psi})(x, 0, D) W_{1}=\left.\left(\partial_{t} \widetilde{\bar{U}}\right)\right|_{t=0}$. Therefore, if $U$ and $\widetilde{U}$ are exact solutions to the initial value problems (10) with the same initial conditions as $\bar{U}$ and $\widetilde{\bar{U}}$, respectively, then $\mathrm{WF}(\bar{U})=\mathrm{WF}(U)$ and $\mathrm{WF}(\widetilde{\bar{U}})=\mathrm{WF}(\widetilde{U})$.

Since $\Lambda_{\rho, \mathbf{C}}=\Lambda_{\widetilde{\rho}, \widetilde{\mathbf{C}}}$, and the initial values of $U$ agree with those of $\widetilde{U}$, we conclude as in Section 3.1 that $U=\widetilde{U}$ on $\left(\mathbb{R}^{3} \backslash \Omega\right) \times[0, T]$. It follows that $\operatorname{WF}(U)=\operatorname{WF}(\widetilde{U})$ on $\left(\mathbb{R}^{3} \backslash \Omega\right) \times[0, T]$, and so

$$
\Gamma^{+}=\widetilde{\Gamma}^{+} \quad \text { and } \quad \Gamma^{-}=\widetilde{\Gamma}^{-} \quad \text { on }\left(\mathbb{R}^{3} \backslash \Omega\right) \times[0, T] .
$$

Hence, for $T$ large enough (so that any disturbance propagating as a third mode wave can pass through $\Omega$ before time $T)$ the bicharacteristics for $(\rho, \mathbf{C})$ and $(\widetilde{\rho}, \widetilde{\mathbf{C}})$ that enter $\Omega$ at the same position, direction, and time must also exit $\Omega$ at the same position, direction, and time. In particular, the time needed for the disturbance to travel through the object is the same for $(\rho, \mathbf{C})$ and $(\widetilde{\rho}, \widetilde{\mathbf{C}})$.

We can vary $x_{0}$ and $\xi_{0}$ (the position and direction of the initial disturbance) arbitrarily to arrive at the conclusion of Theorem 1. In fact, the entire scattering relation (see [59]) is determined.

In the case of transverse isotropy with GWP and a disjoint mode we have that $\lambda_{2}(x, \xi)$ has the form $\xi^{t} g_{2}^{-1} \xi$, and so the canonical projections of the bicharacteristics $\Gamma^{ \pm}, \widetilde{\Gamma}^{ \pm}$onto $\mathbb{R}^{3}$ correspond to geodesic curves with respect to the metrics $g_{2}, \widetilde{g}_{2}$, respectively. (See, for example, [60].) Consequently, the geodesic distance between boundary points corresponds to the travel time along the bicharacteristics, and we conclude that the geodesic distances $d_{g_{2}}, d_{\sigma_{2}}$ with respect to $g_{2}$ and $\widetilde{g}_{2}$ between the entry and exit points agree in the case that the boundary of $\Omega$ is strictly convex with respect to each of $g_{2}$ and $\widetilde{g}_{2}$, for example. (Geodesic distance with respect to a Riemannian metric $g$ is defined as the infimum over all length of geodesics connecting two given points, provided that the manifold $(\Omega, g)$ is complete, that is, provided that any two points on the boundary can be joined by a geodesic. This completeness condition holds if the boundary of $\Omega$ is strictly convex with respect to the metrics, for example.)

In Corollary 3 below, we show that the metric $g_{2}$ is uniquely determined by the Dirichlet-to-Neumann map (up to a natural obstruction) by applying boundary rigidity results, under either of the following additional assumptions 
on the metric:

i) the metrics are close to the Euclidean metric, and satisfy a curvature bound [29], [30]; or

ii) the metrics satisfy a (weaker) curvature bound, but are known to be close to one another [31].

Corollary 3 Suppose that the conditions of Theorem 1 hold, and that one of the conditions (17) that guarantee boundary rigidity holds. Then

implies

$$
\begin{gathered}
\Lambda_{\rho, \mathbf{C}}=\Lambda_{\tilde{\rho}, \tilde{\mathbf{c}}} \\
g_{2}=\psi_{2}^{*} \widetilde{g}_{2}
\end{gathered}
$$

for some diffeomorphism $\psi_{2}$ of $\bar{\Omega}$ that fixes the boundary.

We note, for example, that if the elasticity tensor is of the form (GWP12) or (GWP2iso), then condition (17i) can be satisfied. On the other hand, $g_{2}$ is not generally conformal to the Euclidean metric, so we cannot employ the results in [61], [62]. In [6], we give pointwise conditions under which a transversely isotropic tensor $\mathbf{C}$ is the pull-back of an isotropic tensor via a diffeomorphism. If $\mathbf{C}$ has GWP, then $g_{2}$ can be the pull-back of the Euclidean metric, that is, a flat metric. Gromov [63] showed that flat metrics are boundary rigid. Stefanov and Uhlmann consider real analytic metrics in [28].

On determining the geodesic distances for the modes that are not disjoint. We conclude this section with a few comments on the problem of determining the first two metrics $g_{0}, g_{1}$, whose corresponding wave modes are not disjoint.

We first observe that for transversely isotropic media with GWP and a disjoint mode the characteristic set of the operator I $\partial_{t}^{2}-A_{01}(x, D)$ can be easily described exactly. In fact, it consists of the union $\Sigma=S_{0} \cup S_{1}$, where $S_{i}$ are the first and second-mode light cones $\tau^{2}-\xi^{t} g_{i}^{-1}(x) \xi, i=0,1$, intersecting tangentially exactly along a conic surface $\Sigma_{2}$ the doubly characteristic set.

The doubly characteristic surface $\Sigma_{2}$ can be involutive under certain non-generic conditions. (It always is for coincident modes). In this situation, applying results of Dencker [64], we can conclude that for any initial data the wave front set of the solution of the Cauchy problem for the decoupled operator (12) is invariant under the union of the Hamilton flows of each eigenvalue $\lambda_{i}, i=0,1$, as for systems of real principal type. Then, arguments similar to those in the previous sections give that the Dirichlet-to-Neumann map uniquely determines the wave front set for the first and second wave modes. However, we cannot exclude the possibility that bicharacteristics may become associated with a different wave mode after passing through a point of intersection of the sheets of the light cone, and so the travel time might not be related to the boundary distance function of one particular metric.

For anisotropic elastic media with GWP, one disjoint mode, and two coincident modes, one can resolve the degeneracy of the coincident eigenvalues and corresponding bicharacteristics (all double) and reduce to the case of a $2 \times 2$ system of real principal type with simple eigenvalues and characteristics. This situation arises in isotropic elastodynamics, where there are two coincident shear wave modes and a single compressional wave mode. In this paper, we give the example of transversely isotropic media with two coincident shear modes (corresponding to $\left.g_{0}=g_{1}\right)$ and one quasi-compressional mode (corresponding to $g_{2}$ ), which does not reduce to the isotropic case. See case (GWP2iso) in Section 2.2. The wave front set of the solution $U$ satisfies (see [49, Theorem 2.2, page 154]):

$$
\mathrm{WF}(U) \subset \operatorname{Char}\left(\partial_{t}^{2}-\Delta_{g_{0}}\right) \uplus \operatorname{Char}\left(\partial_{t}^{2}-\Delta_{g_{2}}\right),
$$

and $\operatorname{WF}(U) \cap \operatorname{Char}\left(\partial_{t}^{2}-\Delta_{g_{j}}\right), j=0,2$, is invariant under the Hamilton flow of $\tau^{2}-\xi^{t} g_{j} \xi$. Hence, we can proceed as above to conclude that both metrics are determined up to diffeomorphism. The isotropic case was treated in [13].

Corollary 4 (Determination of the Riemannian metrics for coincident modes) Under the hypotheses of Corollary 3, if $\mu_{T}=\mu_{L}, \widetilde{\mu}_{T}=\widetilde{\mu}_{L}$, and in addition either condition (17i) or (17ii) is satisfied for the metrics $g_{j}$, $\widetilde{g}_{j}, j=0,2$, then there exists a diffeomorphism $\psi_{j}: \Omega \rightarrow \Omega$, with $\psi_{j}\left\llcorner_{\partial \Omega}=I d\right.$, such that:

$$
g_{j}=\psi_{j}^{*} \widetilde{g}_{j}, \quad j=0,2 .
$$




\subsection{Uniqueness of material parameters}

We consider transversely isotropic elastic media $(\Omega, \mathbf{C}, \rho)$ and $(\Omega, \widetilde{\mathbf{C}}, \widetilde{\rho})$ that satisfy the conditions of Corollary 3 , each with a disjoint mode with GWP and associated Riemannian metric $g_{2}$ and $\widetilde{g}_{2}$ (respectively). Let $k, \widetilde{k}$ be unit vectors representing the axes of transverse isotropy (respectively) for $(\Omega, \mathbf{C}, \rho)$ and $(\Omega, \widetilde{\mathbf{C}}, \widetilde{\rho})$. Then the Riemannian metric tensors have the form $g_{2}^{-1}=p I+q(k \otimes k)$ and $\widetilde{g}_{2}^{-1}=\widetilde{p} I+\widetilde{q}(\widetilde{k} \otimes \widetilde{k})$, with $p, q$, $\tilde{p}$, $\widetilde{q}$ scalars. (See Section 2.2.) Let $\psi_{2}$ be the diffeomorphism of $\bar{\Omega}$ associated with $g_{2}$ and $\widetilde{g}_{2}$ under the conditions of Corollary 3 ; that is, $g_{2}=\psi_{2}^{*} \widetilde{g}_{2}$. Then

$$
p I+q(k \otimes k)=\psi_{2}^{*}(\widetilde{p} I+\widetilde{q}(\widetilde{k} \otimes \widetilde{k})) .
$$

We apply Lemma 5, given below, to conclude that if the Dirichlet-to-Neumann maps for the elastic media are the same, then $p$ and $\widetilde{p}$ (and $q$ and $\widetilde{q}$ ) are related via the diffeomorphism $\psi_{2}$. In particular, writing $a^{2}, b^{2}, c^{2}$ for the eigenvalues of $\left[\left(D \psi_{2}\right)^{t}\left(D \psi_{2}\right)\right]^{-1}$, and $V$ for the orthogonal matrix appearing in the polar decomposition of $D \psi_{2}^{-1}=\sqrt{\left[\left(D \psi_{2}\right)^{t}\left(D \psi_{2}\right)\right]^{-1}} V$, we find that

$$
p=a^{2}\left(\widetilde{p} \circ \psi_{2}\right) \quad \text { and } \quad q=\left(\widetilde{q} \circ \psi_{2}\right)\left|\psi_{2}^{*} \widetilde{k}\right|^{2}+\left(\widetilde{p} \circ \psi_{2}\right)\left(c^{2}-a^{2}+b^{2}-a^{2}\right) .
$$

That is, two of the five parameters for transversely isotropic elastodynamics with GWP are determined by the Dirichlet-to-Neumann map, up to composition with $\psi_{2}$, and multiplication by scalars determined by $\psi_{2}$ and $\widetilde{k}_{\text {. }}$

We also conclude from Lemma 5 that (19) imposes certain restrictions on $\psi_{2}$. In fact, at points $x \in \Omega$ where $k$ and $\psi_{2}^{*} \widetilde{k}$ are parallel, $V$ maps $\widetilde{k} \circ \psi_{2}$ onto the line spanned by $k$, i.e., $V\left(\widetilde{k} \circ \psi_{2}\right)= \pm k$. Also, in this case $D \psi_{2}^{-1}$ has a two-dimensional eigenspace with $a^{2}=b^{2}$, and the eigenvalue $c$ of $D \psi_{2}^{-1}$ is given by $|c|=\left|\psi_{2}^{*} \widetilde{k}\right|$. Finally, for any orthogonal unit vectors $k^{\perp}$ and $k_{\perp}$ spanning the space orthogonal to $k$, we have that

$$
D \psi_{2}^{-1}=\left[a\left(k^{\perp} \otimes k^{\perp}+k_{\perp} \otimes k_{\perp}\right)+c(k \otimes k)\right] V .
$$

At points $x \in \Omega$ where $k$ and $\psi_{2}^{*} \widetilde{k}$ are orthogonal, we have that $k^{t} V\left(\widetilde{k} \circ \psi_{2}\right)=0, \quad\left|\psi_{2}^{*} \widetilde{k}\right|^{2}=b^{2}\left[K^{t} V\left(\widetilde{k} \circ \psi_{2}\right)\right]^{2}$, $\left(\widetilde{q} \circ \psi_{2}\right)\left|\psi_{2}^{*} \widetilde{k}\right|^{2}=-\left(\widetilde{p} \circ \psi_{2}\right)\left(b^{2}-a^{2}\right)$, and

$$
D \psi_{2}^{-1}=[a(k \times K) \otimes(k \times K)+b(K \otimes K)+c(k \otimes k)] V
$$

for $K=\left(\psi_{2}^{*} \widetilde{k}\right) /\left|\psi_{2}^{*} \widetilde{k}\right|$. At points $x \in \Omega$ where $k$ and $\psi_{2}^{*} \widetilde{k}$ are not parallel or orthogonal, we have that

$$
D \psi_{2}^{-1}=\left[a(k \times K) \otimes(k \times K)+b\left(u^{+} \otimes u^{+}\right)+c\left(u^{-} \otimes u^{-}\right)\right] V
$$

for $\quad \psi_{2}^{*} \widetilde{k} /\left|\psi_{2}^{*} \widetilde{k}\right|=s k+t K$ (with $s, t$ scalars), $\quad u^{ \pm}=\left[2 \widetilde{q}\left|\psi_{2}^{*} \widetilde{k}\right|^{2} s t\right] k+\left[q-\widetilde{q}\left|\psi_{2}^{*} \widetilde{k}\right|^{2}\left(s^{2}-t^{2}\right) \pm D\right] K$,

$$
K=\frac{k \times\left(k \times \psi_{2}^{*} \widetilde{k}\right)}{\left|k \times\left(k \times \psi_{2}^{*} \tilde{k}\right)\right|}, \quad D=\sqrt{\left[q-\widetilde{q}\left|\psi_{2}^{*} \widetilde{k}\right|^{2}\right]^{2}+4 q \widetilde{q}\left|\psi_{2}^{*} \widetilde{k}\right|^{2} t^{2}}
$$

Example. In the case of (CM1) or (CM2) we have from (7) and (8) that the disjoint mode metric for (GWP1) is $g_{2}^{-1}=\widehat{\mu}_{L} I+\left(\widehat{B}-\widehat{\mu}_{L}\right)(k \otimes k)$, for $\left(\right.$ GWP2) and $($ GWP2iso $)$ is $g_{2}^{-1}=\left(\widehat{A}+\widehat{\mu}_{T}\right) I+\left(\widehat{B}-\widehat{A}-\widehat{\mu}_{T}\right)(k \otimes k)$, while for $(G W P 12)$ is $g_{2}^{-1}=\widehat{\mu}_{L} I$, with $\widehat{\mu}_{T}=\mu_{T} / \rho, \widehat{\mu}_{L}=\mu_{L} / \rho, \widehat{A}=A / \rho, \widehat{B}=B / \rho, \widehat{C}=C / \rho$. We conclude from (20) that $r+R p=\left(\widetilde{r} \circ \psi_{2}\right)\left|\psi_{2}^{*} \widetilde{k}\right|^{2}$, where $r=p+q, R=\left(a^{2}-b^{2}-c^{2}+\left|\psi_{2}^{*} \widetilde{k}\right|^{2}\right) / a^{2}$, and so the following relations among the material parameters hold, where $a^{2}, b^{2}, c^{2}$ are the eigenvalues of $\left[\left(D \psi_{2}\right)^{t}\left(D \psi_{2}\right)\right]^{-1}$ :

$(G W P 1)$

$$
\widehat{\mu}_{L}=a^{2}\left(\widehat{\widetilde{\mu}}_{L}\right) \circ \psi_{2}, \quad \widehat{B}+R \widehat{\mu}_{L}=\left(\widehat{\widetilde{B}} \circ \psi_{2}\right)\left|\psi_{2}^{*} \widetilde{k}\right|^{2},
$$

$(G W P 2), \quad(G W P 2 i s o) \quad \widehat{A}+\widehat{\mu}_{T}=a^{2}\left(\widehat{\widetilde{A}}+\widehat{\widetilde{\mu}}_{T}\right) \circ \psi_{2}, \quad \widehat{B}+R\left(\widehat{A}+\widehat{\mu}_{T}\right)=\left(\widehat{\widetilde{B}} \circ \psi_{2}\right)\left|\psi_{2}^{*} \widetilde{k}\right|^{2}$,

( GWP12)

$$
\widehat{\mu}_{L}=a^{2}\left(\widehat{\widetilde{\mu}}_{L}\right) \circ \psi_{2}, \quad \widehat{\mu}_{L}+R \widehat{\mu}_{L}=\left(\widehat{\widetilde{\mu}}_{L} \circ \psi_{2}\right)\left|\psi_{2}^{*} \widetilde{k}\right|^{2}, \quad \text { so } \quad 2 a^{2}=b^{2}+c^{2}
$$


Lemma 5 Suppose that

$$
p I+q(k \otimes k)=\widetilde{p} M+\widetilde{q}(\widetilde{k} \otimes \widetilde{k}),
$$

where $p, q, \widetilde{p}, \widetilde{q}$ are scalars, $k$ and $\widetilde{k}$ are unit vectors, and $M$ is a symmetric, invertible matrix with eigenvalues $a^{2}, b^{2}, c^{2}$. Then the scalars $p, q, \widetilde{p}, \widetilde{q}$ are related by

$$
p=a^{2} \widetilde{p}, \quad q=\widetilde{q}+\widetilde{p}\left(c^{2}-a^{2}+b^{2}-a^{2}\right) .
$$

In addition, (21) imposes certain restrictions on $M$. In the case that $k$ and $\widetilde{k}$ are parallel, we find that $M$ has a two-dimensional eigenspace with $a^{2}=b^{2}$. In the case that $k$ and $\widetilde{k}$ are orthogonal, we find that $\widetilde{q}=-\widetilde{p}\left(b^{2}-a^{2}\right)$, and the eigenvectors of $a^{2}, b^{2}$, and $c^{2}$ (respectively) are $k \times \widetilde{k}, \widetilde{k}$, and $k$.

Proof. In the case that $k$ and $\widetilde{k}$ are parallel, it follows from (21) that

$$
\widetilde{p} M=p I+[q-\widetilde{q}](k \otimes k)=p\left(k^{\perp} \otimes k^{\perp}+k_{\perp} \otimes k_{\perp}\right)+[p+q-\widetilde{q}](k \otimes k) .
$$

Therefore, up to reordering of the eigenvalues, $p=\widetilde{p} a^{2}, p=\widetilde{p} b^{2}, p+q-\widetilde{q}=\widetilde{p} c^{2}$.

Similarly, in the case that $k$ and $\widetilde{k}$ are orthogonal, we have

$$
\widetilde{p} M=p(k \times \widetilde{k}) \otimes(k \times \widetilde{k})+(p-\widetilde{q})(\widetilde{k} \otimes \widetilde{k})+(p+q)(k \otimes k),
$$

and so (again, up to reordering of the eigenvalues) $p=\widetilde{p} a^{2}, p-\widetilde{q}=\widetilde{p} b^{2}, p+q=\widetilde{p} c^{2}$.

If $k$ and $\widetilde{k}$ are not parallel or orthogonal, then the eigenvalues of $\widetilde{p} M=p I+q(k \otimes k)-\widetilde{q}(\widetilde{k} \otimes \widetilde{k})$ are $\underset{\widetilde{k}}{p}=\widetilde{p} a^{2}, p+\left(q-\widetilde{q}|\widetilde{k}|^{2}\right) / 2+D / 2=\widetilde{p} b^{2}$, and $p+\left(q-\widetilde{q}|\widetilde{k}|^{2}\right) / 2-D / 2=\widetilde{p} c^{2}$, where $K=[k \times(k \times \widetilde{k})] /|k \times(k \times \widetilde{k})|$, $\widetilde{k}=s k+t K$ (with $s, t$ scalars), and $D=\sqrt{(q-\widetilde{q})^{2}+4 q \widetilde{q} t^{2}}$. It follows (since $s^{2}+t^{2}=|\widetilde{k}|^{2}=1$ ) that $p, \widetilde{p}, q, \widetilde{q}$ are related by (22). An eigenvector corresponding to the first eigenvalue of $\widetilde{p} M$ is $k \times \widetilde{k} /|k \times \widetilde{k}|$. Writing the other two eigenvectors as $u^{ \pm}=\alpha^{ \pm} k+\beta^{ \pm} K$, we reduce the formulas for $u^{ \pm}$to

$$
\left(\begin{array}{cc}
-\left[q-\widetilde{q}\left(s^{2}-t^{2}\right) \pm D\right] & 2 \widetilde{q} s t \\
2 \widetilde{q} s t & q-\widetilde{q}\left(s^{2}-t^{2}\right) \pm D
\end{array}\right) \cdot\left(\begin{array}{l}
\alpha^{ \pm} \\
\beta^{ \pm}
\end{array}\right)=\left(\begin{array}{l}
0 \\
0
\end{array}\right) .
$$

It follows that $\widetilde{p} M=\widetilde{p} a^{2}([k \times \widetilde{k} /|k \times \widetilde{k}|] \otimes[k \times \widetilde{k} /|k \times \widetilde{k}|])+\widetilde{p} b^{2}\left(u^{+} \otimes u^{+}\right)+\widetilde{p} c^{2}\left(u^{-} \otimes u^{-}\right)$, with $u^{ \pm}=[2 \widetilde{q} s t] k+\left[q-\widetilde{q}\left(s^{2}-t^{2}\right) \pm D\right] K$.

\section{Appendix: Construction of a smooth extension of $\rho$ and C}

We prove here that we can extend $\rho, \widetilde{\rho}$ and $\mathbf{C}, \widetilde{\mathbf{C}}$ to be smooth on $\mathbb{R}^{3}$, preserving strong ellipticity and the disjoint mode condition, and so that $\rho=\widetilde{\rho}$ and $\mathbf{C}=\widetilde{\mathbf{C}}$ in $\mathbb{R}^{3} \backslash \Omega$, if $\Omega$ is diffeomorphic to a ball.

We begin by assuming as in Theorem 1 that the densities and elasticity tensors agree to infinite order at the boundary of $\Omega$. Since $\rho, \widetilde{\rho}$ and $\mathbf{C}, \widetilde{\mathbf{C}}$ are $C^{\infty}(\bar{\Omega})$, there is some open, collar neighborhood $V$ of $\Omega$ with $\rho, \widetilde{\rho}$ and $\mathbf{C}, \widetilde{\mathbf{C}}$ defined and smooth in $V \cup \bar{\Omega}$. We write $\lambda_{i}(x, \xi), i=1,2,3$, for the eigenvalues of the principal symbol $\mathbf{C}[\cdot, \xi, \cdot, \xi]$ of the elliptic part of $P_{\rho, \mathrm{C}}$ (and, similarly, $\widetilde{\lambda}_{i}(x, \xi)$ for $P_{\widetilde{\rho}, \tilde{\mathbf{C}}}$, and observe that the $\lambda_{i}(x, \xi)$ are continuous in $V \cup \bar{\Omega}$ since the eigenvalues of a square matrix depend continuously on its entries [65, p. 540]; see also [66, p. 812]. The strong ellipticity condition for $\mathbf{C}$ is an open condition on the $\lambda_{i}(x, \xi)$, i.e., $\lambda_{i}(x, \xi)>0$, since $\mathbf{C}$ is strongly elliptic if and only if $\mathbf{C}[\cdot, \xi, \cdot, \xi]$ is positive definite; the disjoint mode condition $\lambda_{2}(x, \xi) \neq \lambda_{i}(x, \xi), i=0,1, x \in \bar{\Omega}, \xi \in \mathbb{R}^{3} \backslash 0$ is also an open condition on the $\lambda_{i}$. Therefore, $\mathbf{C}$ is strongly elliptic and has a distinct eigenvalue $\lambda_{2}$ in $V \cup \bar{\Omega}$, possibly for some smaller, open, collar neighborhood $V$ of $\Omega$. We shrink $V$ if necessary so that $V$ can be represented locally in terms of boundary normal coordinates $\left(\widetilde{x}^{\prime}, \widetilde{x}_{3}\right)$ by $\widetilde{x}_{3} \in[0,1]$ with locally $\partial \Omega=\left\{\widetilde{x}_{3}=0\right\}$ and $\partial(V \cup \bar{\Omega})=\left\{\widetilde{x}_{3}=1\right\}$. Let $\phi\left(\widetilde{x}_{3}\right)$ be a smooth function on $[0,1]$ with $\phi(0)=0, \phi(1)=1, \phi^{(m)}(0)=0$, and $\phi^{(m)}(1)=0, m=1,2, \ldots$ Then, we define a new extension $\mathcal{P}(x)$ of $\rho(x)$ by $\mathcal{P}(x)=\rho(x)$ on $\Omega, \mathcal{P}(x)=\left[1-\phi\left(\widetilde{x}_{3}\right)\right] \rho\left(\widetilde{x}^{\prime}, \widetilde{x}_{3}\right)+\phi\left(\widetilde{x}_{3}\right) M$ in $V$, and $\mathcal{P}(x)=M$ on $\mathbb{R}^{3} \backslash(V \cup \bar{\Omega})$, where $M$ is a positive constant. 
We define a new extension $\mathcal{C}(x, \xi)$ of $\mathbf{C}(x)[\cdot, \xi, \cdot, \xi]$ in a similar fashion. First, we observe that since $\mathbb{R}^{3} \backslash \bar{\Omega}$ is diffeomorphic to the exterior of a ball in $\mathbb{R}^{3}$, it follows that we can construct "polar" coordinates $\left(\widetilde{x}^{\prime}, \widetilde{x}_{3}\right)$ for $\mathbb{R}^{3} \backslash(V \cup \bar{\Omega})$ that smoothly extend the boundary normal coordinates in $V$ to the whole of $\mathbb{R}^{3}$. For $0<\omega \leq 1$ we define $V^{\omega} \subseteq V$ to be the collar neighborhood of $\Omega$ with $0 \leq \widetilde{x}_{3} \leq \omega$, and we define a family of extensions of $\mathbf{C}(x)[\cdot, \xi, \cdot, \xi]$ by

$$
\mathcal{C}(x, \xi, \omega)=\left\{\begin{array}{lr}
\mathbf{C}(x)[\cdot, \xi, \cdot, \xi] & \text { on } \bar{\Omega} \times\left(\mathbb{R}^{3} \backslash 0\right), \\
{\left[1-\phi\left(\frac{\widetilde{x}_{3}}{\omega}\right)\right] \mathbf{C}\left(\widetilde{x}^{\prime}, \widetilde{x}_{3}\right)[\cdot, \xi, \cdot, \xi]+\phi\left(\frac{\widetilde{x}_{3}}{\omega}\right) \mathbf{C}\left(\widetilde{x}^{\prime}, 0\right)[\cdot, \xi, \cdot, \xi]} & \text { in } V^{\omega} \times\left(\mathbb{R}^{3} \backslash 0\right), \\
\mathbf{C}\left(\widetilde{x}^{\prime}, 0\right)[\cdot, \xi, \cdot, \xi] & \text { on }\left(\mathbb{R}^{3} \backslash\left(V^{\omega} \cup \bar{\Omega}\right)\right) \times\left(\mathbb{R}^{3} \backslash 0\right) .
\end{array}\right.
$$

It follows that $\mathcal{C}(x, \xi, \omega)$ is smooth on $\mathbb{R}^{3} \times\left(\mathbb{R}^{3} \backslash 0\right)$ for any $0<\omega \leq 1$ since $\phi$ is flat to infinite order at the boundary of the collar neighborhood $V^{\omega}$. In addition, $\mathcal{C}\left(\widetilde{x}^{\prime}, 0, \xi, 0\right)=\mathbf{C}\left(\widetilde{x}^{\prime}, 0\right)[\cdot, \xi, \cdot, \xi]$ extends $\mathcal{C}(x, \xi, \omega)$ continuously to $\omega=0$ in $\mathcal{V}=\left\{(x, \xi, \omega) \mid 0 \leq \omega \leq 1,0 \leq \widetilde{x}_{3} \leq \omega,\left(\widetilde{x}^{\prime}, 0\right) \in \partial \Omega, \xi \neq 0\right\}$. We denote by $\underline{\lambda}_{i}(x, \xi, \omega)$ the eigenvalues, and $\underline{v}_{i}(x, \xi, \omega)$ corresponding orthonormal eigenvectors, of $\mathcal{C}(x, \xi, \omega)$ in $\mathbb{R}^{3} \times\left(\mathbb{R}^{3} \backslash 0\right) \times[0,1]$; in particular, let $\underline{\lambda}_{2}$ be the eigenvalue that is $\lambda_{2}$ at $\partial \Omega$. Then the $\underline{\lambda}_{i}$ are uniformly continuous in $\mathbb{R}^{3} \times\left(\mathbb{R}^{3} \backslash 0\right) \times[0,1]$ since they are homogeneous of degree 2 in $\xi$ and, as above, they are continuous on the compact domain $\left\{(x, \xi, \omega)|| \xi \mid=1,0 \leq \omega \leq 1,0 \leq \widetilde{x}_{3} \leq \omega,\left(\widetilde{x}^{\prime}, 0\right) \in \partial \Omega\right\}$. It follows, since $\underline{\lambda}_{2}$ differs from $\underline{\lambda}_{i}, i=0,1$, at $\omega=0$, that there is a uniform $\omega_{0}>0$ so that $\underline{\lambda}_{2}$ differs from $\underline{\lambda}_{i}, i=0,1$, for any $0 \leq \widetilde{x}_{3} \leq \omega_{0}$, any $\left(\widetilde{x}^{\prime}, 0\right) \in \partial \Omega$, and any $\xi \neq 0$. Each entry of $\mathcal{C}\left(x, \xi, \omega_{0}\right)$ is a homogeneous polynomial in $\xi$ of order 2 , and is smooth on $T^{*}\left(\mathbb{R}^{3}\right) \backslash 0$. Also, $\mathcal{C}\left(x, \xi, \omega_{0}\right)$ is positive-definite since in $V^{\omega_{0}} \times\left(\mathbb{R}^{3} \backslash 0\right)$ it is the sum of positive-definite terms.

We recover a smooth extension $\mathcal{C}$ of the elasticity tensor $\mathbf{C}$ from $\mathcal{C}\left(x, \xi, \omega_{0}\right)$. In fact, for any elasticity tensor $\mathbf{C}$ and its symbol $\mathbf{C}[\cdot, \xi, \cdot, \xi]$ we have $\mathbf{C}\left[e_{i}, \xi, e_{k}, \xi\right]=\mathrm{C}^{i 1 k 1} \xi_{1}^{2}+2 \mathrm{C}^{i 1 k 2} \xi_{1} \xi_{2}+2 \mathrm{C}^{i 1 k 3} \xi_{1} \xi_{3}+\mathrm{C}^{i 2 k 2} \xi_{2}^{2}+2 \mathrm{C}^{i 2 k 3} \xi_{2} \xi_{3}+\mathrm{C}^{i 3 k 3} \xi_{3}^{2}$ is a homogeneous, degree-2 polynomial in $\xi$, and so $\mathbf{C}^{i j k l}$ is given by the coefficient of the $\xi_{j} \xi_{l}$-term of $e_{i}^{t} \mathbf{C}[\cdot, \xi, \cdot, \xi] e_{k}$ if $j=l$, for example. It follows that the smooth extension $\mathcal{C}$ of $\mathbf{C}$ to $\mathbb{R}^{3}$ preserves strong ellipticity (since $\mathcal{C}\left(x, \xi, \omega_{0}\right)$ is positive-definite) and the disjoint mode condition.

We can modify the smooth extension $\mathcal{C}(x, \xi, \omega)$ of $\mathbf{C}[\cdot, \xi, \cdot, \xi]$ so that the property of geodesic wave propagation (GWP) is preserved for the disjoint mode. Let $\underline{\lambda}_{2}$ be the eigenvalue of $\mathcal{C}(x, \xi, \omega)$ that corresponds to the disjoint mode. Then $\underline{\lambda}_{2}$ is smooth on $\mathbb{R}^{3} \times\left(\mathbb{R}^{3} \backslash 0\right)$ for $0<\omega \leq 1$ by the Implicit Function Theorem. In addition, we have $\underline{\lambda}_{2}=\xi^{t} g_{2}^{-1} \xi$ on $\bar{\Omega}$, so $\left(g_{2}\right)_{i j}^{-1}(x)=\partial_{\xi_{i}} \xi_{j} \underline{\lambda}_{2}(x, \xi, \omega) / 2=\left(\partial_{\xi_{i}} \xi_{j} \underline{\lambda}_{2}\right)\left(x, \xi_{0}, \omega\right) / 2$ on $\bar{\Omega}$ for any $\xi_{0} \neq 0$. We define an extension $\mathcal{G}_{2}$ of $g_{2}$ to $\mathbb{R}^{3}$ for $0<\omega \leq 1$ by $\left(\mathcal{G}_{2}\right)_{i j}^{-1}(x, \omega)=\left(\partial_{\xi_{i}} \xi_{j} \lambda_{2}\right)\left(x, \xi_{0}, \omega\right) / 2$, where $\xi_{0}$ is fixed with $\left|\xi_{0}\right|=1$. Then for $0<\omega \leq 1$ $\mathcal{G}_{2}$ is smooth, symmetric, and positive-definite (since $\xi^{t}\left(\mathcal{G}_{2}\right)^{-1} \xi=\underline{\lambda}_{2}(x, \xi, \omega)>0$ for all $\xi \neq 0$ ) on $\mathbb{R}^{3}$. We modify $\mathcal{C}(x, \xi, \omega)$ so that $\underline{\lambda}_{2}$ is replaced by $\xi^{t} \mathcal{G}_{2}^{-1} \xi$ in $V^{\omega} \times\left(\mathbb{R}^{3} \backslash 0\right)$, and by $\xi^{t} \mathcal{G}_{2}^{-1}\left(\widetilde{x}^{\prime}, 0, \omega\right) \xi$ in $\left(\mathbb{R}^{3} \backslash\left(V^{\omega} \cup \bar{\Omega}\right)\right) \times\left(\mathbb{R}^{3} \backslash 0\right)$ :

$$
\left\{\begin{array}{rr}
\mathbf{C}(x)[\cdot, \xi, \cdot, \xi] & \text { on } \bar{\Omega} \times\left(\mathbb{R}^{3} \backslash 0\right), \\
{\left[1-\phi\left(\frac{\widetilde{x}_{3}}{\omega}\right)\right] \mathbf{C}\left(\widetilde{x}^{\prime}, \widetilde{x}_{3}\right)[\cdot, \xi, \cdot, \xi]+\phi\left(\frac{\widetilde{x}_{3}}{\omega}\right) \mathbf{C}\left(\widetilde{x}^{\prime}, 0\right)[\cdot, \xi, \cdot, \xi]} & \\
+\left(\xi^{t} \mathcal{G}_{2}^{-1} \xi-\underline{\lambda}_{2}(x, \xi, \omega)\right)\left(\underline{v}_{2} \otimes \underline{v}_{2}\right)(x, \xi, \omega) \quad \text { in } V^{\omega} \times\left(\mathbb{R}^{3} \backslash 0\right), \\
\mathbf{C}\left(\widetilde{x}^{\prime}, 0\right)[\cdot, \xi, \cdot, \xi]+\left(\xi^{t} \mathcal{G}_{2}^{-1}\left(\widetilde{x}^{\prime}, 0, \omega\right) \xi-\underline{\lambda}_{2}\left(\widetilde{x}^{\prime}, 0, \xi, \omega\right)\right)\left(\underline{v}_{2} \otimes \underline{v}_{2}\right)\left(\widetilde{x}^{\prime}, 0, \xi, \omega\right) \\
\text { on }\left(\mathbb{R}^{3} \backslash\left(V^{\omega} \cup \bar{\Omega}\right)\right) \times\left(\mathbb{R}^{3} \backslash 0\right) .
\end{array}\right.
$$

This extension is homogeneous of order 2 in $\xi$, is smooth on $\mathbb{R}^{3} \times\left(\mathbb{R}^{3} \backslash 0\right)$ for $0<\omega \leq 1$ (since $\underline{v}_{2}$ is smooth by the Implicit Function Theorem), and is positive-definite (since $\mathcal{G}_{2}$ is). The resulting eigenvalues of (24) are uniformly continuous in $\mathbb{R}^{3} \times\left(\mathbb{R}^{3} \backslash 0\right) \times[0,1]$ as above, and so, since the eigenvalue $\xi^{t}\left(\mathcal{G}_{2}\right)^{-1} \xi$ is disjoint from $\underline{\lambda}_{i}, i=0,1$, at $\omega=0$, we again conclude that, for some $\omega_{0}>0$ small enough, $\xi^{t}\left(\mathcal{G}_{2}\right)^{-1}\left(x, \omega_{0}\right) \xi$ is always different than $\underline{\lambda}_{i}\left(x, \xi, \omega_{0}\right), i=0,1$, on $\mathbb{R}^{3} \times\left(\mathbb{R}^{3} \backslash 0\right)$. 
In the case of transversely isotropic elastic media with GWP we observe that strong ellipticity, geodesic wave propagation, and the property of having a disjoint mode are described in terms of the conditions (6), (GWP1), (GWP2), and (CM1) and (CM2) in Table 1. These conditions on the scalar parameters appearing in the spectral representation of the elasticity tensor are either open conditions, or they define dependent parameters in terms of others that are free to vary. Since the material parameters are smooth in a neighborhood of $\Omega$, we can choose a collar neighborhood $V$ in which the open conditions on the independent parameters hold, and then extend those parameters to the whole of $\mathbb{R}^{3}$ in a fashion similar to the above extension of the density $\rho$. The extension of the dependent parameters follows.

We conclude the extension of the material parameters by setting $\widetilde{\rho}$ equal to $\rho$ and $\widetilde{\mathbf{C}}$ equal to $\mathbf{C}$ outside $\Omega$. It follows that $\widetilde{\rho}$ and $\widetilde{\mathbf{C}}$ are smooth on $\mathbb{R}^{3}$ since by hypothesis $(\widetilde{\rho}, \widetilde{\mathbf{C}})$ agree with $(\rho, \mathbf{C})$ to infinite order at $\partial \Omega$.

\section{Acknowledgments}

The authors thank M. de Hoop, P. Stefanov, and C. Stolk for useful discussions. They would also like to acknowledge the continuous support of their Ph.D. advisers, M.E. Taylor and G. Uhlmann.

\section{References}

[1] G. Uhlmann, editor, Inside out: inverse problems and applications, volume 47 of Mathematical Sciences Research Institute Publications (Cambridge University Press, Cambridge, 2003).

[2] L. Ji, R. McLaughlin, D. Renzi, and J.-R. Yoon, Interior elastodynamics inverse problems: shear wave speed reconstruction in transient elastography, Inverse Problems 19 (6) (2003) S1-S29.

[3] C. C. Stolk and M. V. d. Hoop, Microlocal analysis of seismic inverse scattering in anisotropic elastic media, Comm. Pure Appl. Math. 55 (3) (2002) 261-301.

[4] E. T. Quinto, L. Ehrenpreis, A. Faridani, F. Gonzalez, and E. Grinberg, editors, Radon transforms and tomography, volume 278 of Contemporary Mathematics (American Mathematical Society, Providence, RI, 2001).

[5] J. Trampert, Global seismic tomography: the inverse problem and beyond, Inverse Problems 14 (3) (1998) 371-385.

[6] A. L. Mazzucato and L. Rachele, Partial uniqueness and obstruction to uniqueness in inverse problems for anisotropic elastic media, Accepted.

[7] J. E. Marsden and T. J. R. Hughes, Mathematical foundations of elasticity (Dover Publications, Inc., New York, 1994).

[8] M. J. P. Musgrave, Crystal Acoustics (Holden-Day, 1970).

[9] K. H. Hellwege, H. Landolt, and R. Börnstein, Table 11, Elastic constants for the hexagonal system, and Table 13, Elastic constants for the hexagonal system (non-crystalline materials), in: Zahlenwerte und Funktionen aus Naturwissenschaften und Technik [Numerical data and functional relationships in science and technology], Neue Serie, volume III/11 (Berlin, Springer-Verlag, 1961-) 39-49.

[10] A. Mazzucato and L. Rachele, On transversely isotropic elastic media with ellipsoidal slowness surfaces, In preparation.

[11] J. Sylvester and G. Uhlmann, Inverse problems in anisotropic media, in: Inverse scattering and applications (Amherst, MA, 1990), volume 122 of Contemp. Math. (Amer. Math. Soc., Providence, RI, 1991) 105-117.

[12] L. V. Rachele, Boundary determination for an inverse problem in elastodynamics, Comm. Partial Differential Equations 25 (11-12) (2000) 1951-1996.

[13] L. V. Rachele, Uniqueness in inverse problems for elastic media with residual stress, Comm. Partial Differential Equations 28 (11-12) (2003) 1787-1806.

[14] L. V. Rachele, Uniqueness of the density in an inverse problem for isotropic elastodynamics, Trans. Amer. Math. Soc. 355 (12) (2003) 4781-4806. 
[15] L. V. Rachele, Uniqueness in inverse problems for elastic media with residual stress, Comm. Partial Differential Equations 28 (11-12) (2003) 1787-1806.

[16] S. Hansen and G. Uhlmann, Propagation of polarization in elastodynamics with residual stress and travel times, Math. Ann. 326 (3) (2003) 563-587.

[17] R. L. Robertson, Boundary identifiability of residual stress via the Dirichlet to Neumann map, Inverse Problems 13 (4) (1997) 1107-1119.

[18] R. L. Robertson, Determining residual stress from boundary measurements: a linearized approach, J. Elasticity $\mathbf{5 2}$ (1) (1998) 63-73.

[19] C.-S. Man, Hartig's law and linear elasticity with initial stress, Inverse Problems 14 (2) (1998) 313-319.

[20] G. Nakamura and J.-N. Wang, Unique continuation for an elasticity system with residual stress and its applications, SIAM J. Math. Anal. 35 (2) (2003) 304-317.

[21] V. Isakov, G. Nakamura, and J.-N. Wang, Uniqueness and stability in the Cauchy problem for the elasticity system with residual stress, in: Inverse problems: theory and applications (Cortona/Pisa, 2002), volume 333 of Contemp. Math. (Amer. Math. Soc., Providence, RI, 2003) 99-113.

[22] C.-L. Lin, Strong unique continuation for an elasticity system with residual stress, Indiana Univ. Math. J. 53 (2) (2004) 557-582.

[23] C.-L. Lin and J.-N. Wang, Uniqueness in inverse problems for an elasticity system with residual stress by a single measurement, Inverse Problems 19 (4) (2003) 807-820.

[24] S. A. Ivanov, C.-S. Man, and G. Nakamura, Recovery of residual stress in a vertically heterogeneous elastic medium, IMA J. Appl. Math. 70 (1) (2005) 129-146.

[25] A. Hoger, On the residual stress possible in an elastic body with material symmetry, Arch. Rational Mech. Anal. 88 (3) (1985) 271-289.

[26] A. Hoger, On the determination of residual stress in an elastic body, J. Elasticity 16 (3) (1986) 303-324.

[27] G. Nakamura, K. Tanuma, and G. Uhlmann, Layer stripping for a transversely isotropic elastic medium, SIAM J. Appl. Math. 59 (5) (1999) 1879-1891.

[28] P. Stefanov and G. Uhlmann, Boundary rigidity and stability for generic simple metrics, J. Amer. Math. Soc. 18 (4) (2005) 975-1003.

[29] P. Stefanov and G. Uhlmann, Rigidity for metrics with the same lengths of geodesics, Math. Res. Lett. 5 (1-2) (1998) 83-96.

[30] M. Lassas, V. Sharafutdinov, and G. Uhlmann, Semiglobal boundary rigidity for Riemannian metrics, Math. Ann. 325 (4) (2003) 767-793.

[31] C. B. Croke, N. S. Dairbekov, and V. A. Sharafutdinov, Local boundary rigidity of a compact Riemannian manifold with curvature bounded above, Trans. Amer. Math. Soc. 352 (9) (2000) 3937-3956.

[32] H. Rund, The differential geometry of Finsler spaces, in: Die Grundlehren der Mathematischen Wissenschaften, Bd. 101 (Springer-Verlag, Berlin, 1959).

[33] D. Bao, S.-S. Chern, and Z. Shen, An introduction to Riemann-Finsler geometry, volume 200 of Graduate Texts in Mathematics (Springer-Verlag, New York, 2000).

[34] P. L. Antonelli, A. Bóna, and M. A. Slawiński, Seismic rays as Finsler geodesics, Nonlinear Anal. Real World Appl. 4 (5) (2003) 711-722.

[35] M. P. Rudzki, Parametric representation of the elastic wave in anisotropic media, Journal of Applied Geophysics 54 (3-4) (2003) 165-183.

[36] K. Helbig, Foundations of anisotropy for exploration seismics (Pergamon, 1994).

[37] K. Helbig and L. Thomsen, 75-plus years of anisotropy in exploration and reservoir seismics: A historical review of concepts and methods, Geophysics 70 (6)November-December (2005) 9ND-23ND.

[38] R. G. Payton, Classification of wave front shapes for transversely isotropic elastic media, Ist. Lombardo Accad. Sci. Lett. Rend. A 108 (1974) 684-698. 
[39] P. Chadwick and A. N. Norris, Conditions under which the slowness surface of an anisotropic elastic material is the union of aligned ellipsoids, Quart. J. Mech. Appl. Math. 43 (4) (1990) 589-603.

[40] R. Burridge, P. Chadwick, and A. N. Norris, Fundamental elastodynamic solutions for anisotropic media with ellipsoidal slowness surfaces, Proc. Roy. Soc. London Ser. A 440 (1910) (1993) 655-681.

[41] P. M. Bakker, About the completeness of the classification of cases of elliptic anistropy, Proc. Roy. Soc. London Ser. A 451 (1942) (1995) 367-373.

[42] P. F. Daley and L. R. Lines, Linearized quantities in transversely isotropic media, Can. J. Earth Sci 41 (3) (2004) 349-354.

[43] L. Pestov and G. Uhlmann, Two dimensional compact simple Riemannian manifolds are boundary distance rigid, Ann. of Math. (2) 161 (2) (2005) 1093-1110.

[44] M. E. Taylor, Reflection of singularities of solutions to systems of differential equations, Comm. Pure Appl. Math. 28 (4) (1975) 457-478.

[45] P. J. Braam and J. J. Duistermaat, Normal forms of real symmetric systems with multiplicity, Indag. Math. (N.S.) 4 (4) (1993) 407-421.

[46] C. Nolan and G. Uhlmann, Parametrices for symmetric systems with multiplicity, Preprint.

[47] L. Hörmander, The calculus of Fourier integral operators, in: Prospects in mathematics (Proc. Sympos., Princeton Univ., Princeton, N.J., 1970), Ann. of Math. Studies, No. 70 (Princeton Univ. Press, Princeton, N.J., 1971) $33-57$.

[48] J. J. Duistermaat, Fourier integral operators (Courant Institute of Mathematical Sciences, New York University, New York, 1973).

[49] M. E. Taylor, Pseudodifferential operators, volume 34 of Princeton Mathematical Series (Princeton University Press, Princeton, N.J., 1981).

[50] N. Dencker, On the propagation of polarization sets for systems of real principal type, J. Funct. Anal. 46 (3) (1982) 351-372.

[51] G. Duvaut and J.-L. Lions, Inequalities in mechanics and physics, volume 219 of Grundlehren der Mathematischen Wissenschaften (Springer-Verlag, Berlin, 1976).

[52] J. Merodio and R. W. Ogden, A note on strong ellipticity for transversely isotropic linearly elastic solids, Quart. J. Mech. Appl. Math. 56 (4) (2003) 589-591.

[53] P. Chadwick, Wave propagation in transversely isotropic elastic media. I. Homogeneous plane waves, Proc. Roy. Soc. London Ser. A 422 (1862) (1989) 23-66.

[54] M. E. Taylor, Partial differential equations. II. Qualitative studies of linear equations, volume 116 of Applied Mathematical Sciences (Springer-Verlag, New York, 1996).

[55] C. Gérard, Propagation de la polarisation pour des problèmes aux limites convexes pour les bicaractéristiques, Comm. Partial Differential Equations 10 (12) (1985) 1347-1382.

[56] L. Hörmander, The analysis of linear partial differential operators. I. Distribution theory and Fourier analysis, volume 256 of Grundlehren der Mathematischen Wissenschaften [Fundamental Principles of Mathematical Sciences] (Springer-Verlag, Berlin, 1983).

[57] Y. V. Egorov, Linear differential equations of principal type, in: Contemporary Soviet Mathematics (Consultants Bureau, New York, 1986).

[58] L. Hörmander, The analysis of linear partial differential operators. III. Pseudo-differential operators, volume 274 of Grundlehren der Mathematischen Wissenschaften [Fundamental Principles of Mathematical Sciences] (Springer-Verlag, Berlin, 1994).

[59] V. Guillemin, Sojourn times and asymptotic properties of the scattering matrix, in: Proceedings of the Oji Seminar on Algebraic Analysis and the RIMS Symposium on Algebraic Analysis, volume 12 (1976/77 supplement), (Kyoto Univ., Kyoto, 1976) 69-88.

[60] V. I. Smirnov, A course of higher mathematics. Vol. IV [Integral equations and partial differential equations] (Pergamon Press, Oxford, 1964). 
[61] R. G. Muhometov, On a problem of reconstructing Riemannian metrics, Sibirsk. Mat. Zh. 22 (3) (1981) 119-135, 237(English translation: Siberian Math. J. 22 (3) (1982) 420-433).

[62] R. G. Muhometov and V. G. Romanov, On the problem of finding an isotropic Riemannian metric in an $n$ dimensional space, Dokl. Akad. Nauk SSSR 243 (1) (1978) 41-44(English translation: Soviet Math. Dokl. 19 (6) (1979) 1330-1333).

[63] M. Gromov, Filling Riemannian manifolds, J. Differential Geom. 18 (1) (1983) 1-147.

[64] N. Dencker, On the propagation of singularities for pseudo-differential operators with characteristics of variable multiplicity, Comm. Partial Differential Equations 17 (9-10) (1992) 1709-1736.

[65] R. A. Horn and C. R. Johnson, Matrix analysis (Cambridge University Press, Cambridge, 1990).

[66] L. Dieci and T. Eirola, On smooth decompositions of matrices, SIAM J. Matrix Anal. Appl. 20 (3) (1999) 800-819. 